\title{
Symmetry aspects in stability investigations for thin membranes
}

\author{
Anders Eriksson $^{1}$ • Arne Nordmark ${ }^{1}$
}

Received: 28 February 2016 / Accepted: 2 July 2016 / Published online: 1 August 2016

(C) The Author(s) 2016. This article is published with open access at Springerlink.com

\begin{abstract}
Modelling of structural instability problems is considered for thin square membranes subjected to hydrostatic pressure, with a focus on the effects from symmetry conditions considered or neglected in the model. An analysis is performed through group-theoretical concepts of the symmetry aspects present in a flat membrane with one-sided pressure loading. The response of the membrane is described by its inherent differential eigensolutions, which are shown to be of five different types with respect to symmetry. A discussion is given on how boundary conditions must be introduced in order to catch all types of eigensolutions when modelling only a subdomain of the whole. Lacking symmetry in a FEM model of the whole domain is seen as a perturbation to the problem, and is shown to affect the calculated instability response, hiding or modifying instability modes. Numerical simulations verify and illustrate the analytical results, and further show the convergence with mesh fineness of different aspects of instability results.
\end{abstract}

Keywords Bifurcation · Symmetry-breaking · Group theory $\cdot$ Meshing $\cdot$ Boundary conditions

\section{Introduction}

Symmetry in structures is often a result from aesthetic considerations or from functional optimization. For instance, many biological structures possess a high degree of symmetry. It is well known that the optimized structural forms are very sensitive to all kinds of imperfections. This paper aims to discuss

Anders Eriksson

anderi@kth.se

1 KTH Mechanics, Royal Institute of Technology, Osquars Backe 18, 10044 Stockholm, Sweden how considered or neglected symmetries in the modelling of a problem significantly can affect the computational results.

When analyzing a structure, it is tempting to utilize an existing symmetry for improved efficiency or accuracy, and symmetry has been utilized in many common solution methods. Perhaps most prominent in classical analyses for plates and shells, the consideration of odd and even functions in series solutions was an important aspect [42]. It was also earlier an important competence for structural analysts to be able to split general loadings into symmetric and antisymmetric subcases, and this topic has been an important part of engineering curricula [13, and many others].

The symmetry considerations have also been important aspects of the development of finite element methods ('FEM'). In particular, this was the case in earlier days, when computer resources were commonly limiting the analyses, and such simplifications were necessary for obtaining results with maximum accuracy $[33,45]$.

Even if the above methods occasionally used also diagonal symmetry sections in a Cartesian system, for instance in the analysis of $\frac{1}{8}$ of a square plate, most traditional symmetry considerations have considered mirror symmetries (and, occasionally, anti-symmetries) with respect to the three principal coordinate planes, by introducing essential boundary conditions of zero displacements. Similar reasoning has guided analyses of circular domains by extracting a repetitive sector of the full geometry.

Analytical treatments of the symmetry aspects of square and circular pressurized membranes are very similar. The present work focusses on a square domain, based on two arguments. First, it is believed that the square domain is the one for which an engineer would be most confident in using the obvious mirror symmetries in the axis planes, and it is therefore important to point to the effects from this assumption. The second reason is that the square membrane affected 
by a pressure has a somewhat richer set of instabilities, due to the differences between the side and diagonal symmetry planes. For the present context, the corners of the square domain do not lead to any particular problems.

The above symmetry assumptions have been based on an identification of a symmetric model geometry affected by symmetric loading and other boundary conditions. In a linear static analysis, this typically implies that the calculated response will fulfill the same symmetry conditions. But, even if the linear static response of a structure may show the considered symmetry conditions, dynamic as well as instability responses do not necessarily inherit these. Well-known examples are vibration and buckling modes of rectangular plates, but also the full dynamic response of a simply supported beam or frame, where the unsymmetries in the simple supports can affect results through the mass distribution. A general recommendation has commonly been one of caution in using symmetry simplifications in these situations.

The present work considers the symmetry and regularity aspects in the context of instability analyses of thin pressurized membranes with a highly symmetric shape. This class of simulations is valid for a large variety of thin threedimensional inflatable structures used in several engineering and medical contexts $[23,26,29]$. These situations are often both geometrically non-linear due to finite deformations and materially non-linear through the constitutive relationship. Analytical results can be obtained for several simple geometries [28,35], and numerical treatments are also available $[3,4]$. Several accompanying aspects are important, such as load descriptions [16], contacts [1,21], and dynamics [15], but also instabilities of many forms: limit and turning solutions, bifurcations, and wrinkling.

The thin membranes are often modelled as hyper-elastic, with several different formulations, each with a number of free parameters. Several studies have shown that the relations between the constitutive parameters in a material model significantly affect the response of simulated membranes $[11,30]$. The Mooney-Rivlin material model $[27,34]$ is frequently shown to produce reasonably good approximation of stresses in a material at least for moderate strains, and is computationally convenient $[5,18]$. The materials can be affected by instability at certain stress states; such instabilities are discussed in literature [17,20,24, and many others].

The most prominent instability phenomena in thin membranes are, however, related to the geometric non-linearity, where significant configuration changes result from pressurization. For a fluid-loaded membrane, different stability conclusions are reached when a fluid level or a fluid volume is controlled [43]. Bifurcations, for instance symmetrybreaking deformation modes can also occur in the loading process. Wrinkling, corresponding to more or less localized domains of compressive stresses also often occur for these problems [36,39], and can be seen as related to geometric effects. Overall instability of a structure due to a consideration of wrinkling through a relaxed energy formulation [32] or the tension-field theory [38], is shown in [31]. General aspects of, primarily, geometric instabilities are discussed in classical works $[22,41]$, but also often in the context of finite element simulations [2, and others].

The current paper first gives a brief review of the numerical modelling of thin membranes used for the experiments below. Then, a description is given of the symmetry aspects relevant for the considered square structure and its FEM discretization. An analytical treatment shows how a subdomain modelling can hide or modify instabilities, and how the domain symmetry and accompanying sets of boundary conditions for equilibrium solution and eigenmode extraction affect instability representation and conclusions. A set of illustrative numerical simulations are given to demonstrate the relevance of the analytical conclusions. A few concluding remarks are given on the effects from symmetry in the modelling of instability affected structures.

\section{Mechanical modelling}

Sophisticated simulation algorithms are needed for the evaluation and interpretation of instability in a general FEM-based context. A reliable finite element formulation is needed, which is not overly sensitive to, e.g., the scaling of the problem. A solution algorithm must also be used, which can isolate and identify the critical situations.

\subsection{Structural model}

As shell models are less reliable for membrane analyses, and are also computationally demanding, simulations of the loading process have been based purely on the membrane behavior in the present work. The mechanical model was thereby one of local plane stress conditions, but in a 3D setting. Triangular elements with linear kinematic assumptions were used for discretization of the membrane [11]. In brief, the element formulation is based on a Total Lagrangian form, assuming the element to keep flat in all configurations. Strains and stresses are constant within the element. Pre-stressing of the structure was introduced by prescribing displacements of edge nodes, with an accompanying change in thickness.

An isotropic incompressible Mooney-Rivlin model was used, and based on a strain energy formulation

$W=W(\mathbf{C})=c_{1} I_{1}(\mathbf{C})+c_{2} I_{2}(\mathbf{C})$,

where $I_{1}$ and $I_{2}$ are the two first invariants of the right Cauchy-Green deformation tensor $\mathrm{C}$. The constitutive constants were related to a shear modulus $\mu$ and a 'hardening 
parameter' $k$, according to:

$c_{1}=\frac{1}{2} \frac{\mu}{1+k}, \quad c_{2}=k c_{1}$

Local plane stress conditions and incompressibility, through the third invariant of $\mathrm{C}$ [18], were built into the formulation, giving explicit constitutive relations.

Wrinkling is perhaps the most common instability in thin membranes. Relaxed strain energy forms [32], leading to a tension-field theory [38], are common ways to treat these local effects from compressive stresses, but these can lead to global instability in a simulation model when pressure acts normal to a wrinkled mid-surface [31]. As wrinkling effects are strongly dependent on a used discretized model, wrinkling conditions were here indicated from local stretch values at each equilibrium solution found, and calculations were discontinued. Contacts with hard or soft surrounding surfaces and self-contacts between different parts of the membrane also frequently occur [40], but were verified not to affect the results below.

The kinematic assumptions and the material model were used to formulate the structural internal forces completely from the global structural displacement components $\mathbf{d}$ as:

$\mathbf{f}=\mathbf{f}(\mathbf{d})$

Both $\mathbf{d}$ and $\mathbf{f}$ are $N$-dimensional vectors, with $N$ the number of considered degrees of freedom in the discretized model.

\subsection{Pressure loading}

The loading on a pressurized membrane comes from onesided gas or fluid over-pressures, with significant differences in their formulations. An over-pressure from gas $\psi$ is uniform over all elements, giving the displacement-dependent structural external force vector $\mathbf{p}=\mathbf{p}(\mathbf{d}, \psi)$. A hydro-static pressure, however, often gives a richer and more interesting instability behavior, in particular when the pressure is acting from below on a horizontal membrane [43]. Assuming gravity in the global negative $Z$ direction, the pressure on an element surface is described by $\psi(z)=\rho g\left(Z_{\text {fluid }}-z\right)$ for $z \leq Z_{\text {fluid, }}$, with $\rho$ the fluid density, $g$ the gravitational acceleration, and $Z_{\text {fluid }}$ the fluid surface level; $z$ is the spatial position of a point. Consistent nodal loads then gives a vector of external forces $\mathbf{p}=\mathbf{p}\left(\mathbf{d}, Z_{\text {fluid }}\right)$. In either case of pressure load, the $N$-dimensional vector of global external forces are described by one primary load parameter

$\mathbf{p}=\mathbf{p}(\mathbf{d}, \gamma)$

with $\gamma \equiv \psi$ or $\gamma \equiv Z_{\text {fluid }}$, respectively.

\subsection{Simulation algorithms}

For a quasi-static formulation, the discretized equilibrium between external and internal forces demands solutions satisfying the structural residual equation:

$\mathbf{F}(\mathbf{z}) \equiv \mathbf{f}(\mathbf{d})-\mathbf{p}(\mathbf{d}, \gamma)=\mathbf{0}$

where also $\mathbf{F}$ and $\mathbf{p}$ are of dimension $N$, and $\mathbf{z}$ is an $(N+1)$ dimensional vector collecting all variables:

$\mathbf{z}=\left(\mathbf{d}^{\mathrm{T}}, \gamma\right)^{\mathrm{T}}$

This system defines one-dimensional curve segments, which are represented by a sequence of equilibrium solutions $\mathbf{z}_{i},(i=0,1, \ldots)$. One particular equilibrium solution is controlled by an extra equation, which can be interpreted as the specification of one mechanical variable, or seen as a numerical branch progress control. In the latter case, the choice of branch control function $[6,12]$, and step-lengths [10], are important aspects of equilibrium branch following.

The variation of the residual expression in Eq. (5) is:

$\delta \mathbf{F}=\left(\mathrm{K}_{d}-\mathrm{K}_{p}\right) \delta \mathbf{d}-\delta \gamma \mathbf{p}_{, \gamma}$

where a subindex following a comma denotes a differential. The expression gives the tangent stiffness matrix,

$\mathrm{K}=\mathrm{K}_{d}-\mathrm{K}_{p}$

including a load-dependent term [37]. Important properties of an equilibrium solution are described by this matrix.

Different forms of instabilities along these branches are also detected and classified. In particular, bifurcations and limit solutions are fundamental for interpreting structural response and stability.

\subsection{Static stability}

Stability is an important aspect of loaded structural systems, and can in broad terms be described in terms of the capacity of the structure to handle disturbances from an established equilibrium configuration, or as a 'power to recover equilibrium'. In this sense, the engineering concept of 'static stability' refers to transitions between static and dynamic behavior, and is a property of a particular static equilibrium configuration. Particular interest is thereby always directed towards changes of the stability when modifying the load parameter, with a critical solution between these domains as the most interesting result. The basis for this viewpoint is the minimum of the total potential energy for a particular load parameter. 
This view on stability is a simplification, and the stability of the structure should rather be seen in a dynamical context, where an introduced kinematic perturbation from a stable equilibrium configuration of the structure will be limited over time, and in particular that the deviation from the static equilibrium can be kept below a specified magnitude by limiting the perturbation.

The stability of an equilibrium configuration is intimately connected to the eigensolutions of the problem. For the discretized model, the eigenvalues of the current tangential stiffness matrix, $\mathrm{K}$, or of the combination of this matrix with a corresponding mass matrix $\mathrm{M}$ lead to the stability conclusions, with the eigenvalues and the eigenvectors. It is noted that, as long as the mass matrix $M$ is positive definite, the eigenvalues of the matrix $\mathrm{K}$ and those of the $(\mathrm{K}, \mathrm{M})$ pair will have the same sign distributions, even if the values are different and dependent on the mass distribution considered.

In the quasi-static setting, with only the $\mathrm{K}$ matrix established and an implicit assumption that mass is equally distributed on all nodes, the eigenvalues are thereby seen as the current principal stiffness values, related to a set of discretized orthonormal displacement vectors. With all eigenvalues positive, the structure is seen as stable, as the potential energy is minimized at this load parameter, and external work is needed to perturb the equilibrium in any specified direction. A zero eigenvalue indicates that a small perturbation in a specific direction can occur at constant potential energy. The present numerical work has only considered the tangent stiffness matrix in the eigenvalue extraction, but it has been previously noted that at least a simplified mass matrix must be introduced when considering constrained equilibrium formulations resulting from, e.g., two-parameter load descriptions [44].

\subsection{Parameter dependence}

For membranes, a key issue is the analysis of the parameter dependence in a simulated response. For this, specially designed algorithms can introduce auxiliary variables, representing a parameterization of the structure or the loading, and then solve the equilibrium problem, and its corresponding stability properties in the higher-dimensional space. The generalized branch-following algorithm used in the present work is discussed in [8]. These formulations use $N_{\gamma}$ parameters $\boldsymbol{\Gamma}$ in the solution set $\mathbf{z}^{\mathrm{T}}=\left[\mathbf{d}^{\mathrm{T}}, \boldsymbol{\Gamma}^{\mathrm{T}}\right]$, and an extended set of equilibrium equations:

$\mathbf{G}(\mathbf{z})=\left[\begin{array}{l}\mathbf{F}(\mathbf{z}) \\ \mathbf{g}(\mathbf{z})\end{array}\right]=\mathbf{0}$

where $\mathbf{g}(\mathbf{z})$ is a set of $N_{g}$ equations, reflecting additional conditions on the sought equilibrium solutions. A typical setting would be to use $N_{g}=N_{\gamma}-1$ control equations for the spec- ification of the physical problem, and one more equation for the numerical placement of each solution on a generalized equilibrium branch. The setting in Eq. (9) allows for very robust and systematic solution of the non-linear equilibrium branches, but has also been used to immediately find critical equilibrium states [7], and to follow the bounds for the feasible region in an optimization setting [9], without the need to solve several complete load-response branches.

\subsection{Symmetry aspects of model and mesh}

Symmetry is seemingly well-known in structural modelling, with a geometrical basis in the sense that a figure or domain is repeated, often in the form of a mirrored copy. In this sense, it has a relation to repetition, where several subdomains of a whole are identical, and simulations can be simplified by only considering only one subdomain with suitable boundary conditions. This can give all equilibrium solutions fulfilling these conditions. The same subdomain and boundary conditions also gives eigenmodes which fulfill these symmetry conditions, showing the bifurcations and the secondary paths emanating at them. With other boundary conditions on the same subdomain, other solutions, bifurcations and secondary paths will be found. In order to find all aspects of instability for a given structure, several sets of boundary conditions may be needed for the solutions, and for the eigenmode calculations. The eigenmodes can thereby be situated in other solution spaces than the equilibrium solution itself, somewhat like the situation appearing for a bar, buckling into a bending response due to a purely compressive force. The symmetry aspects, and their effects on both solutions and eigenmodes, will be further discussed below.

The present work has also considered another symmetry aspect in the discrete FEM-based meshing of the whole domain. The meshing can keep or destroy an inherent symmetry, and can be seen as a perturbation to the continuous model. A set of meshes have allowed different classes of solutions. In particular, some instability modes of a structure can be hidden or otherwise affected by the symmetry of the mesh used.

\subsection{Considered domains and meshes}

The present work primarily focussed on the highly symmetric domain of a square in the $X-Y$-plane. The main characteristic features of the studied problem are its shape, and the fluid pressure loading, which is not symmetric in the direction of gravity, Fig. 1.

The studies of discretizations of a a square domain thereby used a set of subdomains, but also a representative set of meshes, fulfilling different symmetry properties present in the whole domain, Fig. 2. The subdomains, which are grayshaded in the figure, were denoted according to ' $\mathrm{gX}$ ', and 


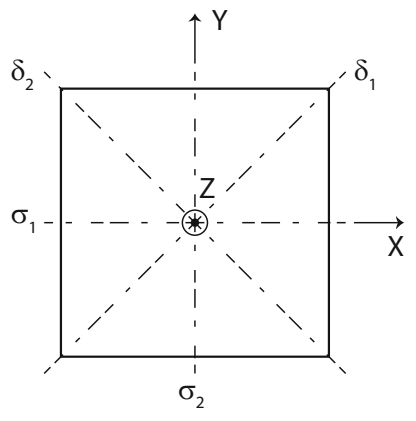

Fig. 1 Geometry of studied square membrane problem, with symmetry aspects marked. Material coordinates, with fluid pressure acting in positive $Z$ direction. Notation for symmetry planes $\sigma_{1}, \sigma_{2}, \delta_{1}, \delta_{2}$ is further discussed below

the meshes according to ' $\mathrm{mX}(\mathrm{nnn})$ ', where ' $\mathrm{mX}$ ' denotes the basic mesh form, and ' $n n n$ ' the number of triangular elements in the mesh. Finer meshes were always created by successively dividing each triangle into four by introducing mid-points on each element edge. For presentation purposes, the mid-point of the square was always introduced as one nodal point.

The subdomains and meshes in Fig. 2 have the following symmetry properties, with further discussion of the terminology below.

Mesh m1 (and subdomain g1): has 4 mirror planes, and repeats when rotating $2 \pi / 4$.

Mesh m2: 2 mirror planes $\left(\sigma_{1}, \sigma_{2}\right)$; minimal rotation $2 \pi / 2$.

Mesh m3: 2 mirror planes $\left(\delta_{1}, \delta_{2}\right)$; rotation $2 \pi / 2$.

Mesh m4: no mirror planes; rotation $2 \pi / 4$.

Mesh m5: no mirror planes; rotation $2 \pi / 2$.

Mesh m6: 1 mirror plane $\left(\sigma_{2}\right)$; rotation $2 \pi / 1$.

Mesh m7: 1 mirror plane $\left(\delta_{1}\right)$; rotation $2 \pi / 1$.

Mesh m8: no mirror planes; rotation $2 \pi / 1$.

Mesh mH (and gH): one half of the whole, which can be completed either by mirroring in $\sigma_{1}$ or by rotating $2 \pi / 2$.

Mesh $\mathbf{m D}$ (and $\mathbf{g D}$ ): one half: mirror in $\delta_{1}$ or rotation $2 \pi / 2$.

Mesh mQ (and gQ): one quarter: successive mirrors in $\sigma_{1}, \sigma_{2}$, or successive rotations $2 \pi / 4$.

Mesh mT (and gT): one quarter: successive mirrors in $\delta_{1}, \delta_{2}$, or successive rotations $2 \pi / 4$.

Mesh mO (and gO): one eighth: successive mirrors in $\sigma_{1}, \delta_{1}$.

\section{Analytical treatment}

This section shows how symmetry can be taken advantage of when finding equilibrium solutions and their stability and bifurcations. This applies both to a continuous model and to

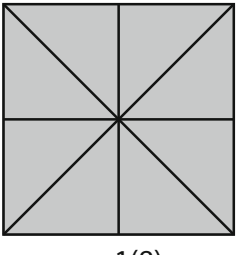

$\mathrm{m} 1(8)$

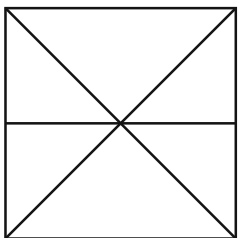

$\mathrm{m} 2(6)$

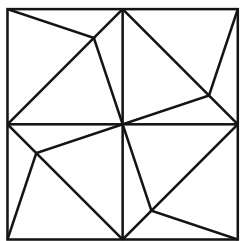

$\mathrm{m} 4(16)$

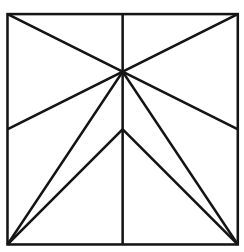

m6(10)

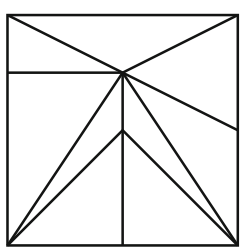

$\mathrm{m} 8(9)$

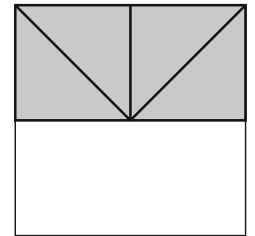

$\mathrm{mH}(4)$

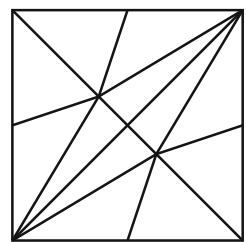

$\mathrm{m} 3(12)$

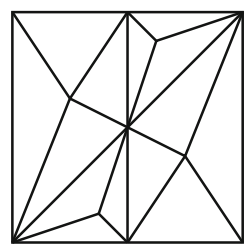

$\mathrm{m} 5(14)$

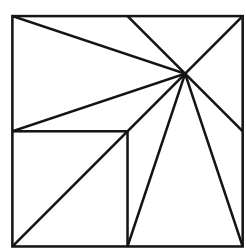

$\mathrm{m} 7(10)$

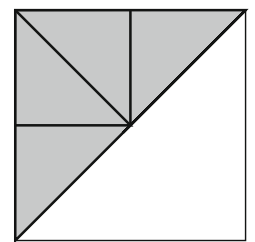

$\mathrm{mD}(4)$

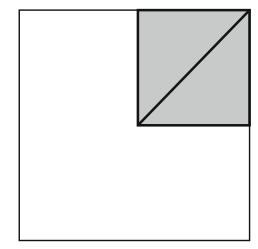

$\mathrm{mQ}(2)$

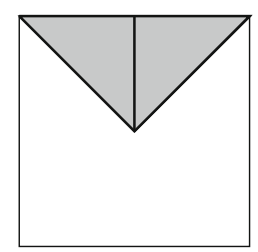

$\mathrm{mT}(2)$

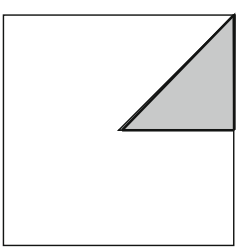

$\mathrm{mO}(1)$
Fig. 2 Symmetry cases used in examples. Basic meshes with number of elements in parenthesis. Meshes are successively refined by dividing each element into four by placing new nodes on element edges, giving meshes of the same type, but with varying element sizes and numbers. Gray areas in figures denote a modelled subdomain, with further discussion below. These subdomains are denoted according to ' $\mathrm{gX}$ ', following the mesh definition ' $\mathrm{mX}$ ' in the figures

a discretized simulation model. The analysis is performed by using group theory concepts $[19,46]$, but differs in its application. While the first reference develops the general theory, and applies this to the symmetry properties of polygon space trusses, the present work focusses on a continuous domain, which is discretized into a finite element mesh. In relation to the element discretization, two main situations are investigated. Firstly, it is for such problem settings tempting to use a 
subdomain of the full problem geometry together with a symmetry group to reconstruct a solution for the full geometry. While computationally efficient, however, this idea restricts the set of solutions that are possible to find. Secondly, discretizing a continuous model might act as a perturbation to the problem, similar to minor changes of the problem geometry, which may lower the symmetry of the problem. Even if the present treatment discusses a rather specific problem, namely the non-linear response of a thin membrane to hydro-static pressure loading, the results are relevant for all non-linear simulations on plane square domains.

In this context, the reader is reminded that a symmetry group is a group where each element is an isometric transformation of 3D space or fields in 3D space. For a finite sized object, the symmetry group is a point group, where each transformation leaves a point fixed. The composition rule for the group is that $a b$ means doing the $b$ transformation first, followed by the $a$ transformation, and the result is another transformation in the group.

\subsection{The symmetry group $C_{4 v}$}

The fluid loaded square membrane has the symmetry group of a square pyramid, $C_{4 v}$. The group consists of the four rotations $r_{0}, r_{1}, r_{2}$, and $r_{3}$ about the positive $z$-axis by $0,1 / 4,1 / 2$, and $3 / 4$ of a full turn, which is counter-clockwise when seen in a common $x$-y-plane view. Additionally, four mirrors $s_{0}, s_{1}, s_{2}$, and $s_{3}$ exist in the $y=0, x=$ $y, x=0$, and $x=-y$ planes. These planes will be denoted $\sigma_{1}, \delta_{1}, \sigma_{2}$, and $\delta_{2}$, respectively, and are shown in Fig. 1. The element $r_{0}$ is the identity element of the group. As an abstract group, $C_{4 v}$ is the dihedral group with 8 elements, so the composition rules are $r_{j} r_{k}=$ $r_{j+k \bmod 4}, r_{j} s_{k}=s_{j+k \bmod 4}, s_{j} r_{k}=s_{j-k \bmod 4}$, and $s_{j} s_{k}=$

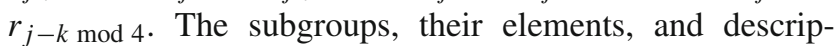
tions of their symmetry properties are shown in Table 1, together with a pointer to the subdomains $\mathrm{gX}$ in Fig. 2 which can be used together with the subgroup to represent the full geometry. As the subgroups constitute a hierarchy, pointers to the next lower subgroups are also given.

\subsection{Using subdomains to find equilibrium solutions}

Equilibrium solutions do not in general inherit the full symmetry $C_{4 v}$ of the problem itself, even if this is the case for solutions on the fundamental branch. Solutions outside this branch may be characterized by any subgroup, down to the completely unsymmetric $C_{1}$.

The solution for a subdomain, together with a matching symmetry subgroup, Table 1 , can be used to reconstruct a solution on the full domain if correct boundary conditions are imposed when obtaining it. The reconstructed solution will necessarily show at least the symmetry of the subgroup used in reconstruction, which means that equilibrium solution branches of lower symmetry are unreachable.

As an example, the subdomain of class ' $\mathrm{gH}$ ', Fig. 2, can be used together with the $C_{1 v}\left(\sigma_{1}\right)$ subgroup. The nonidentity element $s_{0}$ is thereby used to reconstruct the full solution. To ensure continuity of the membrane, the $x$ and $z$ deformed positions must be symmetric about the $\sigma_{1}$ plane. Only equilibrium solutions that obey the $C_{1 v}\left(\sigma_{1}\right)$ or higher $\left(C_{2 v}(\sigma), C_{4 v}\right)$ symmetries can be found this way, while all potential solutions outside this, e.g., any solutions with the mid-point moving in the $y$ direction will be hidden. If, instead the same subdomain of class ' $\mathrm{gH}$ ' is combined with the $C_{2}$ subgroup, continuity requires that $(x, y, z)$ at a point on $\sigma_{1}$ must be equal to $(-x,-y, z)$ at the point rotated by the rotation $r_{2}$, i.e., half a turn. This is thus a non-local boundary condition except at the center point, where the condition is $x=y=0$. Now, reconstructed solutions are restricted to have $C_{2}, C_{2 v}(\sigma), C_{2 v}(\delta), C_{4}$, or $C_{4 v}$ symmetry, which is a different restriction to the full solution space. Both
Table 1 Subgroups of $C_{4 v}$, their elements and a description of the symmetry properties

\begin{tabular}{lllll}
\hline Subgroup & Elements & Description & Subdomain & Children \\
\hline$C_{4 v}$ & $r_{0}, r_{1}, r_{2}, r_{3}$ & $1 / 4$ rotation and all mirrors & $\mathrm{gO}$ & $C_{2 v}(\sigma), C_{4}, C_{2 v}(\delta)$ \\
& $\underline{s_{0}}, \underline{s_{1}}, s_{2}, s_{3}$ & & \\
$C_{4}$ & $r_{0}, \underline{r_{1}}, r_{2}, r_{3}$ & $1 / 4$ rotation and no mirrors & $\mathrm{gQ}, \mathrm{gT}$ & $C_{2}$ \\
$C_{2 v}(\sigma)$ & $r_{0}, r_{2}, \underline{s_{0}}, \underline{s_{2}}$ & Mirrors in $\sigma$ planes & $\mathrm{gQ}$ & $C_{1 v}\left(\sigma_{1}\right), C_{1 v}\left(\sigma_{2}\right), C_{2}$ \\
$C_{2 v}(\delta)$ & $r_{0}, r_{2}, \underline{s_{1}}, \underline{s_{3}}$ & Mirrors in $\delta$ planes & $\mathrm{gT}$ & $C_{1 v}\left(\delta_{1}\right), C_{1 v}\left(\delta_{2}\right), C_{2}$ \\
$C_{2}$ & $r_{0}, \underline{r_{2}}$ & $1 / 2$ rotation and no mirrors & $\mathrm{gH}, \mathrm{gD}$ & $C_{1}$ \\
$C_{1 v}\left(\sigma_{1}\right)$ & $r_{0}, \underline{s_{0}}$ & Mirror in the $\sigma_{1}$ plane & $\mathrm{gH}$ & $C_{1}$ \\
$C_{1 v}\left(\delta_{1}\right)$ & $r_{0}, \underline{s_{1}}$ & Mirror in the $\delta_{1}$ plane & $\mathrm{gD}$ & $C_{1}$ \\
$C_{1 v}\left(\sigma_{2}\right)$ & $r_{0}, \underline{s_{2}}$ & Mirror in the $\sigma_{2}$ plane & & $C_{1}$ \\
$C_{1 v}\left(\delta_{2}\right)$ & $r_{0}, \underline{s_{3}}$ & Mirror in the $\delta_{2}$ plane & & $C_{1}$ \\
$C_{1}$ & $r_{0}$ & Trivial (no) symmetry & $\mathrm{g} 1$ & - \\
\hline
\end{tabular}

The fourth column states which subdomains can be completed by the element. 'Children' denote the next lower groups of symmetry in the hierarchy. Underlined elements are generators of the subgroup 
approaches are able to reconstruct solutions with $C_{2 v}(\sigma)$ or $C_{4 v}$ symmetry, but each approach contains some solutions not found in the other, and neither approach can find solutions with $C_{1}, C_{1 v}\left(\delta_{1}\right), C_{1 v}\left(\sigma_{2}\right)$, or $C_{1 v}\left(\delta_{2}\right)$ symmetry. The combination of subdomain and subgroup, and its associated boundary conditions thereby give the limits for reachable solutions. The numerical simulations below further demonstrate the consequences of this.

\subsection{Representations of $C_{4 v}$ and eigenmodes}

The stability of an equilibrium solution can most often be determined by linear stability analysis. Only when a linear stability analysis finds eigenvalues equal to zero, a non-linear analysis is needed to determine stability. The space of linearized deviations from an equilibrium solution is equal to the space spanned by the complete set of eigenmodes of the solution. The non-zero eigenvalues and their eigenmode shapes depend on what inertia properties are assumed for the problem, but linear stability or instability, i.e., the number of negative eigenvalues is independent of the way mass is introduced, as long as non-zero mass is given to all parts of the structure. Thus, a uniform mass distribution was assumed here for the continuous model, whereas equal node masses were used for the discretized model.

The symmetry of the equilibrium solution will carry over to the problem of finding eigenmodes. Since the eigenmode problem is a linear problem, the elements of the symmetry group will act as linear operators on the space spanned by the eigenmodes, according to group representation theory, previously developed and applied in [19]. A result is that the eigenspace for a problem with finite symmetry group can be written as a direct sum of finite-dimensional spaces, each corresponding to a certain type of representation. Finding these representations is a non-trivial task, but the result is known for the $C_{4 v}$ group and its subgroups. In the following, an equilibrium solution with $C_{4 v}$ symmetry is assumed. The corresponding results for equilibrium solutions with lower symmetry are simpler, but will not be presented in this paper.

Given an equilibrium solution with $C_{4 v}$ symmetry, group representation theory states that the eigenspace can be written as a direct sum of two- or one-dimensional subspaces of the following five types:

I A two-dimensional subspace. We have chosen a basis consisting of one eigenmode $\phi_{1}$ symmetric under the $C_{1 v}\left(\sigma_{1}\right)$ subgroup, and one eigenmode that is the first rotated by $1 / 4$ turn: $\phi_{2}=r_{1} \phi_{1}$. In general, eigenmodes in this space $u \phi_{1}+v \phi_{2}$ have only $C_{1}$ symmetry, unless $u=0, v=0$ or $|u|=|v|$, in which case a $C_{1 v}$ symmetry is present.

II A one-dimensional subspace with one basis vector $\phi$ symmetric under the $C_{2 v}(\sigma)$ subgroup.
III A one-dimensional subspace with one basis vector $\phi$ symmetric under the $C_{2 v}(\delta)$ subgroup.

IV A one-dimensional subspace with one basis vector $\phi$ symmetric under the $C_{4}$ subgroup.

$\mathrm{V}$ A one-dimensional subspace with one basis vector $\phi$ symmetric under the $C_{4 v}$ subgroup.

Examples of eigenmodes of the different types, shown on a deformed membrane configuration can be seen in Fig. 3.

For each of the infinite number of eigenvalues, the corresponding eigenspace will be one of the above types, and thus each eigensolution may be labelled as one of the five types, with eigenvalues of type I double, and the other ones single. These properties of the eigenvalues are independent of whether the model is discretized or not, as long as the discretization preserves the $C_{4 v}$ symmetry of the problem, and thereby has equilibrium solutions with $C_{4 v}$ symmetry.

The eigenvalues of an equilibrium solution will define the stiffness properties of each eigenmode direction, or the second order variation of potential energy when following this mode direction from the equilibrium configuration. In general, these linearized deviations are described by $u \phi_{1}+v \phi_{2}$ for type I and $u \phi$ for the other cases. Representation theory shows how these deviations are affected by the action of each group element, Table 2. For example, the rotation $r_{3}$ acting on an eigenmode of type $\mathbf{I}$ transforms a general direction $[u, v]^{T}$ into the direction described by $[v,-u]^{T}$. As a further example, all mirror elements will change the coefficient $u$ of an eigenmode of type IV to $-u$, i.e., the $u \phi$ is then anti-symmetric with respect to the mirrors.

\subsection{Eigenvalue boundary conditions for subdomains}

Table 2 can be used to identify how boundary conditions can be introduced on eigenmodes to include some of the types and exclude others. For example, using the subdomain ' $\mathrm{gQ}$ ' and enforcing symmetry for an eigenmode on the $\sigma_{1}$ plane and anti-symmetry on the $\sigma_{2}$ plane, means that an eigenmode must be unchanged by the action of the $s_{0}$ group element and must change sign under the action of the $s_{2}$ element. Considering eigenmodes of types II, III, IV, or V, looking at the $s_{0}$ column of Table 2 , the first condition forces $u=-u$, i.e., $u=0$ for types III and IV, whereas the second condition by the $s_{2}$ column forces $u=0$ for types II and $\mathbf{V}$. Thus, no non-zero eigenmode of types II-V can fulfill both these boundary conditions. Considering eigenmodes of type $\mathbf{I}$, both columns $s_{0}$ and $s_{2}$ require $v=0$ with no restrictions on $u$. Any eigenmode evaluated using these boundary conditions must therefore be of type $\mathbf{I}$ and have the form $u \phi_{1}$.

Using for subdomain ' $\mathrm{gQ}$ ' instead symmetry on both planes, meaning that the eigenmode must be unchanged by the action of both $s_{0}$ and $s_{2}$, the new conditions become: $\{u=u, v=-v\},\{u=-u, v=v\}$ for type $\mathbf{I},\{u=u\}$, 
I:

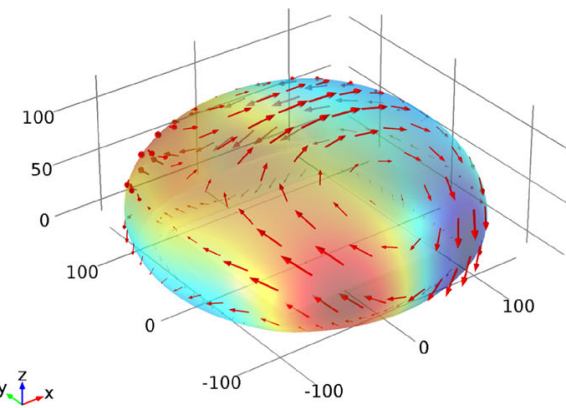

II:

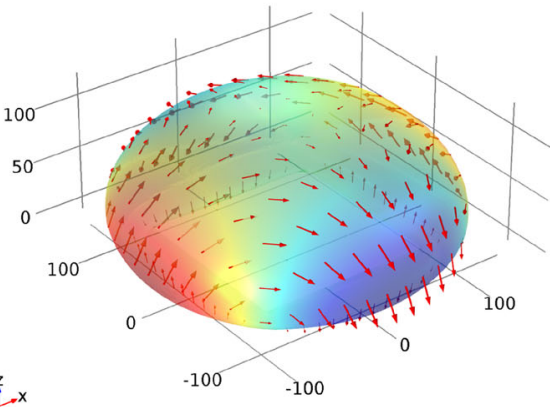

III:

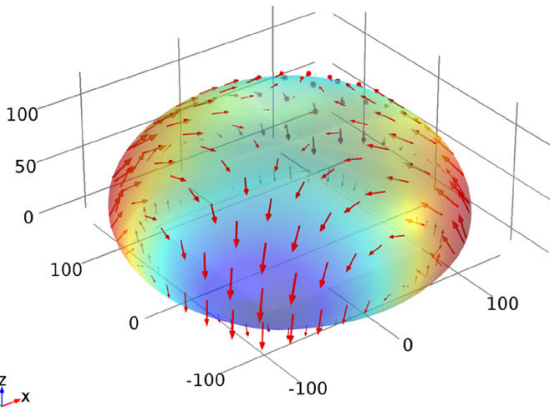

IV:

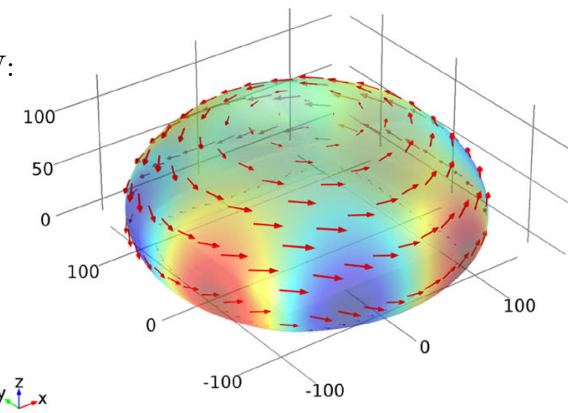

$\mathrm{V}$ :

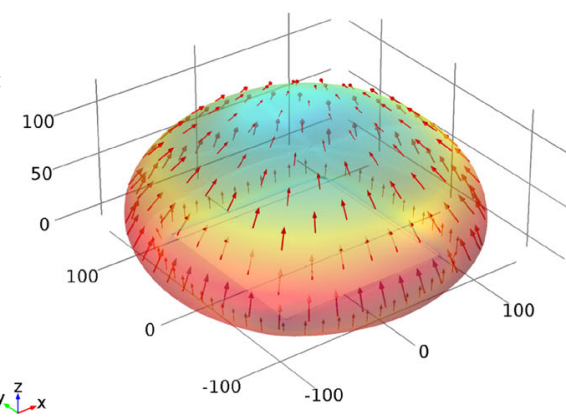

Fig. 3 Example eigenmodes of types $\mathbf{I}-\mathbf{V}$, indicated by arrows, Additionally, the $z$ component is shown by coloring. For type I, eigenmode $\phi_{1}$ is shown in the figure. All modes are plotted on a deflected shape corresponding to the third bifurcation, cf. the numerical solution below $\{u=u\}$ for types II and $\mathbf{V}$, and $\{u=-u\},\{u=-u\}$ for types III and IV. Eigenmodes $u \phi$ of types II or V, but no others, are thus compatible with these boundary conditions.

A systematic usage of boundary conditions in the eigenmode evaluations can thereby often give the complete set of eigenmodes. For the subdomain ' $\mathrm{gQ}$ ' and a $C_{2 v}$ symmetry, an introduction of symmetry conditions on edges $\sigma_{1}$ and $\sigma_{2}$ will admit types $\mathbf{I I}$ and $\mathbf{V}$, whereas anti-symmetric conditions will give types III and IV. Symmetry on one of the edges and anti-symmetry on the other will admit the two types I. Four simulations, with these conditions successively introduced, will thereby facilitate the evaluation of the fundamental solution branch, and admit the solution of the eigenmodes for the same sets of boundary conditions, as all types of modes are reachable from at least one of the simulations.

Similar reasoning concerning symmetry conditions and the eigenspace types for subdomain 'gO', Figs. 1, 2, leads to Table 3. It is noted that these boundary conditions only apply for the eigenmodes, whereas the fundamental solution can be obtained by imposing symmetry on both edges $\sigma_{1}$ and $\delta_{1}$, which only will give type $\mathbf{V}$ limit solutions immediately. For this subdomain choice, all eigenmodes can not be calculated with the same boundary conditions as are valid for the equilibrium solutions. Both eigenmodes must thus be solved for simultaneously for type $\mathbf{I}$, since the modes couple through the boundary condition at $\delta_{1}$. A complete set of eigensolutions can thus be obtained by solving the eigenvalue problem on the smaller domain through four solutions with different simple boundary conditions on the model edges, and then one solution for both types coupled. In the discretized version of this case, the latter case needs a solution vector of double size.

\subsection{Bifurcation of type I for a conservative system}

In relation to the studied problem, the first bifurcation, which corresponds to a double vanishing eigenvalue, type $\mathbf{I}$, is of particular interest, and this will be a main focus for the numerical experiments below. This equilibrium situation will be analyzed here, presenting a heuristic view on the work by Ikeda et al. [19].

For a critical equilibrium solution with $C_{4 v}$ symmetry, and a double zero eigenvalue of type $\mathbf{I}$, a two-dimensional invariant center manifold tangent to the zero eigenspace exists. The original equilibrium solution is represented by $u=v=0$. The two coordinates transform as eigenmode amplitudes, Table 2. Since the system is conservative, a potential function $V(u, v)$ gives the response. The function must be invariant under all transformations of $C_{4 v}$, and the only second order term is $u^{2}+v^{2}$, as all other terms will not be invariant under $s_{0}(u \rightsquigarrow-u, v \rightsquigarrow v)$ and $s_{1}(u \rightsquigarrow v, v \rightsquigarrow u)$, which together generate $C_{4 v}$, according to Table 2. Similar consideration of the fourth order terms, leads to, e.g., the independent func- 
Table 2 The five types of eigenomde representations of $C_{4 v}$

\begin{tabular}{|c|c|c|c|c|c|c|c|c|c|c|}
\hline Type & & & $r_{0}$ & $r_{1}$ & $r_{2}$ & $r_{3}$ & $s_{0}$ & $s_{1}$ & $s_{2}$ & $s_{3}$ \\
\hline I & {$[u, v]$} & $\rightsquigarrow$ & {$[u, v]$} & {$[-v, u]$} & {$[-u,-v]$} & {$[v,-u]$} & {$[u,-v]$} & {$[v, u]$} & {$[-u, v]$} & {$[-v,-u]$} \\
\hline II & $u$ & $\rightsquigarrow$ & $\mathrm{u}$ & $-\mathrm{u}$ & $\mathrm{u}$ & $-\mathrm{u}$ & $\mathrm{u}$ & $-\mathrm{u}$ & $\mathrm{u}$ & $-\mathrm{u}$ \\
\hline III & $u$ & $\rightsquigarrow$ & $\mathrm{u}$ & $-\mathrm{u}$ & $\mathrm{u}$ & $-\mathrm{u}$ & $-\mathrm{u}$ & $\mathrm{u}$ & $-\mathrm{u}$ & $\mathrm{u}$ \\
\hline IV & $u$ & $\rightsquigarrow$ & $\mathrm{u}$ & $\mathrm{u}$ & $\mathrm{u}$ & $\mathrm{u}$ & $-\mathrm{u}$ & $-\mathrm{u}$ & $-\mathrm{u}$ & $-\mathrm{u}$ \\
\hline $\mathbf{V}$ & $u$ & $\rightsquigarrow$ & $\mathrm{u}$ & $\mathrm{u}$ & $\mathrm{u}$ & $\mathrm{u}$ & $\mathrm{u}$ & $\mathrm{u}$ & $\mathrm{u}$ & $\mathrm{u}$ \\
\hline
\end{tabular}

Effects of the elements in the group on the eigenmode amplitudes
Table 3 Boundary conditions for solving all the five eigenmode types on the subdomain ' $\mathrm{gO}$ ' between $y=0$ and $y=x$, Figs. 1, 2

\begin{tabular}{lll}
\hline Type & $\mathrm{BC}$ at $\sigma_{1}$ & $\mathrm{BC}$ at $\delta_{1}$ \\
\hline I & $\phi_{1}: \mathrm{S}, \phi_{2}: \mathrm{AS}$ & $\left(\phi_{1}+\phi_{2}\right): \mathrm{S},\left(\phi_{1}-\phi_{2}\right): \mathrm{AS}$ \\
II & $\phi: \mathrm{S}$ & $\phi: \mathrm{AS}$ \\
III & $\phi:$ AS & $\phi: \mathrm{S}$ \\
IV & $\phi:$ AS & $\phi:$ AS \\
V & $\phi: \mathrm{S}$ & $\phi: \mathrm{S}$ \\
\hline
\end{tabular}

The notation ' $\mathrm{S}$ ' denotes symmetric conditions, 'AS' anti-symmetric. The type I solves the two vectors simultaneously, with both individual and combined boundary conditions

tional terms $\left(u^{2}+v^{2}\right)^{2}$ and $u^{4}-6 u^{2} v^{2}+v^{4}$ as one choice of additions. In polar coordinates, the considered terms are $r^{2}, r^{4}$ and $r^{4} \cos (4 \theta)$. Since both eigenvalues of the symmetric second derivative matrix are assumed to vanish at the critical solution, the second order term must vanish, and the potential can be written as $V(r, \theta)=r^{4}(A+B \cos (4 \theta)) / 24$ up to fourth order, with $A, B$ as situation specific constants.

Assuming there is one parameter in the system which changes the eigenvalues of the $C_{4 v}$ symmetric equilibrium solution, a load parameter term can be added to the potential, as:

$V(r, \theta, \mu)=-\mu r^{2} / 2+r^{4}(A+B \cos (4 \theta)) / 24$,

With the parameter chosen such that an increasing load factor $\mu$ changes the equilibrium solution from stable to unstable, the potential shows positive eigenvalues for $\mu<0$ and negative for $\mu>0$ at $u=v=0$.

With $B \neq 0$, and $|A| \neq|B|$, vanishing of the first differential of the potential in Eq. (10) defines five intersecting curves in the $(u, v, \mu)$ space. Eigenvalues $\lambda$ are obtained from the second differentials of the potential at the equilibrium solution. The results show the following for the branches:

- one branch with $u=v=0$ and double eigenvalues $\lambda_{1,2}=-\mu$. This fundamental branch maintains $C_{4 v}$ symmetry.

- two secondary branches with $\mu=r^{2}(A+B) / 6$ and $\cos (4 \theta)=1$. The eigenvalue corresponding to a vector along the branch is varying like $\lambda_{1}=r^{2}(A+B) / 3$, while the eigenvalue perpendicularly varies as $\lambda_{2}=-2 r^{2} B / 3$. The two branches have $C_{1 v}\left(\sigma_{1}\right)$ and $C_{1 v}\left(\sigma_{2}\right)$ symmetry, respectively, but are equivalent in other respects.

- two secondary branches with $\mu=r^{2}(A-B) / 6$ and $\cos (4 \theta)=-1$. Similarly, the eigenvalues vary as $\lambda_{1}=$ $r^{2}(A-B) / 3$ and $\lambda_{2}=2 r^{2} B / 3$. The branches have $C_{1 v}\left(\delta_{1}\right)$ and $C_{1 v}\left(\delta_{2}\right)$ symmetry, respectively, but are equivalent in other respects.

A local view on the branches passing through the type I bifurcation solution is shown by an example in Fig. 4a, where the symmetry properties of the solutions are indicated. The signs of the relevant eigenvalues are also shown on the branches. Dependent on the bifurcation considered, i.e., the values of $A$ and $B$, the curves might also be turned upwards.

As the scales of $r$ and $\mu$ are not known, only the sign of $A$ and the value of the quotient $A / B$ are important for the description of the bifurcation studied, and these constants can be determined experimentally. For example, if both the secondary branches exist for negative $\mu$ values, then $A<$ 0 and $|B|<|A|$. Then, both branches are unstable, with one or two negative eigenvalues, respectively. The quotients $\lambda_{1} / \lambda_{2}$ on the branches associated with $\sigma$ or $\delta$ symmetry also determine the relative strengths of the axi-symmetric term associated with $A$ in relation to the axi-symmetry-breaking term associated with $B$, since

$$
\begin{aligned}
& \frac{\lambda_{1}}{\lambda_{2}}(\sigma)=-\frac{1}{2}\left(\frac{A}{B}+1\right), \\
& \frac{\lambda_{1}}{\lambda_{2}}(\delta)=\frac{1}{2}\left(\frac{A}{B}-1\right) .
\end{aligned}
$$

For the numerical example studied below, with its chosen parameters, the coefficients are shown to be related as $A / B \approx$ 2.5, with $A<0$, cf. 4.4.

\subsection{Breaking the type I bifurcation by a perturbation of lower symmetry}

The previous section discussed the ideal situation with full symmetry, but in numerical computations, the model is typically discretized, for instance by a finite element mesh. This can be seen as a perturbation to the continuous model, which 


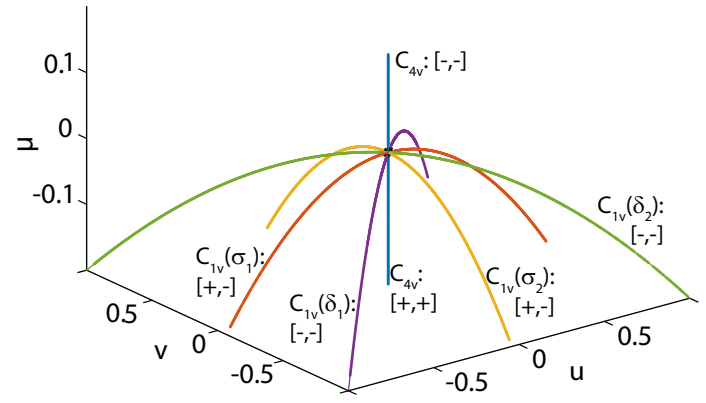

(a)

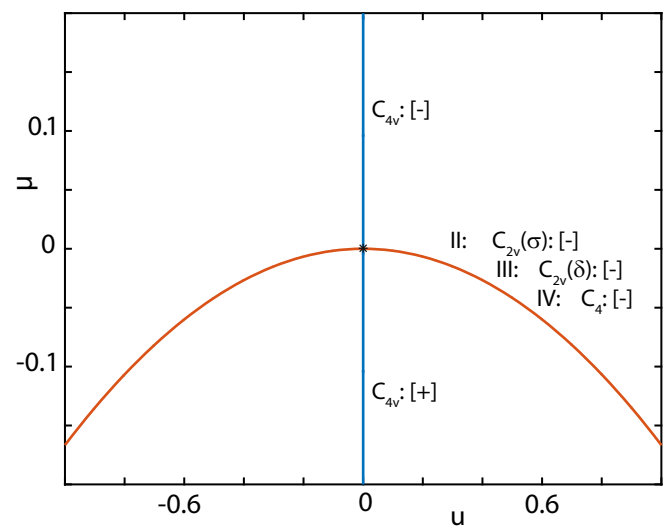

(b)

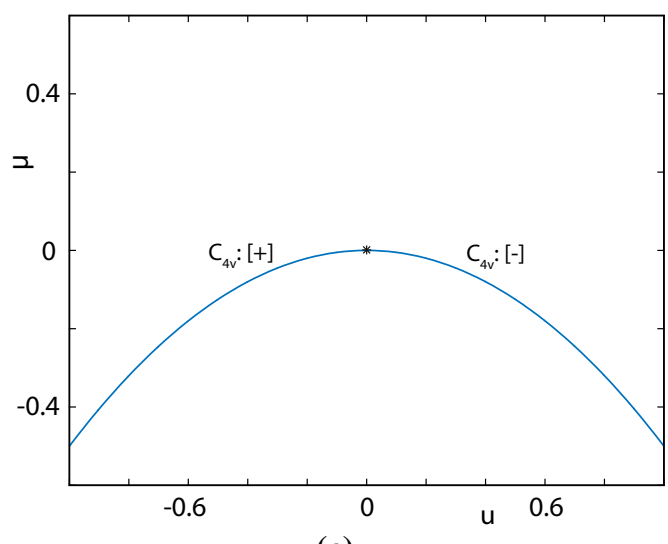

(c)

Fig. 4 Example of equilibrium solution branches passing through a bifurcation, the symmetry properties and the signs of the relevant eigenvalues on the branches. a type I bifurcation; b type II, III and IV bifurcations; c limit solution of type $\mathbf{V} . u$ and $v$ are eigenmode amplitudes, $\mu$ the load parameter. Constants used are $A=-1, B=-0.4$ in $\mathbf{a}, A=-1$ in $\mathbf{b}$ and $\mathbf{c}$. Labels on curves show the symmetry of the solutions, and the signs of the relevant eigenvalues

can reduce the symmetry. This perturbation can be described by a scalar size $\epsilon$, depending on the fineness of the discretization and the order of the numerical method. For finite elements with linear interpolation, $\epsilon \sim h^{2}$ where $h$ is a representative element length. When the discretization only has the symmetry of a subgroup of $C_{4 v}$, additional low order terms with a magnitude proportional to $\epsilon$ will be added to the potential in Eq. (10). Tables 1 and 2 then show which terms are invariant under the different subgroups. An analysis of the lowest order terms which will appear in the potential as a consequence of perturbations of certain classes is summarized in Table 4, cf. the discussion of the possible polynomial terms in 3.5 .

The new terms will affect the response, in the sense that the five branches of an unperturbed bifurcation will be shifted in the load parameter direction by the $r^{2}$ term, and/or broken up by the $\theta$ dependent terms to another critical response. The main effects for the problem studied are that:

- perturbations with 4-fold rotation symmetry $C_{4 v}$ or $C_{4}$ shift the bifurcation in the $\mu$ direction proportional to $\epsilon$, but the double bifurcation remains. For $C_{4}$, the lateral branches are slightly rotated through a next order term $b r^{4} \sin (4 \theta) / 24$, and have only $C_{1}$ symmetry;

- for perturbations with 2-fold rotation symmetry $C_{2 v}$, or $C_{2}$, the central branch $u=v=0$ remains. The non-axisymmetric terms of order $r^{2}$ in $V$ will cause the double zero eigenvalue bifurcation to break. From a balance of term sizes, new bifurcations and/or limit solutions when $\mu \sim \epsilon, r \sim \sqrt{\epsilon}$ are expected to appear.

- for perturbations with no rotation symmetry $C_{1 v}$ or $C_{1}$, the central branch $u=v=0$ will be replaced. The non-axi-symmetric terms of order $r^{1}$ in $V$ will cause the double zero eigenvalue bifurcation to break. New bifurcations and limit solutions will appear when $\mu \sim \epsilon^{2 / 3}, r \sim$ $\epsilon^{1 / 3}$.

The full analyses for two cases are given in Appendix. Exampes of the effects from just the lowest order terms added to the potential in Eq. (10) are illustrated in Fig. 5, for $A$ and $B$ constants close to the ones valid for the numerical example, and arbitrarily chosen perturbation constants $a, b$. Corresponding figures for all the other perturbations are shown together with the numerical simulations below.

\subsection{Bifurcations of types II, III and IV in a conservative system}

The main problem studied will after the first double bifurcation of type I show two single bifurcations of types II and III before a limit solution (type $\mathbf{V}$ ) in the fluid level parameter is reached.

For an equilibrium solution with $C_{4 v}$ symmetry, which has a single zero eigenvalue of type II, III, or IV, a coordinate $u$ is introduced in the one-dimensional center manifold, which transforms as the eigenmode amplitude. Including again a bifurcation parameter $\mu$, the potential will to fourth order be

$V(u, \mu)=-\mu u^{2} / 2+A u^{4} / 24$ 
Table 4 Lowest order additional terms to the potential in Eq. (10), introduced by a small perturbation of lower symmetry

\begin{tabular}{llc}
\hline Symmetry of perturbation & Lowest order terms & Mesh class \\
\hline$C_{4 v}$ & $a r^{2} / 2$ & $\mathrm{~m} 1$ \\
$C_{4}$ & $a r^{2} / 2$ & $\mathrm{~m} 4$ \\
$C_{2 v}(\sigma)$ & $a r^{2} / 2, b r^{2} \cos (2 \theta) / 2$ & $\mathrm{~m} 2$ \\
$C_{2 v}(\delta)$ & $a r^{2} / 2, b r^{2} \sin (2 \theta) / 2$ & $\mathrm{~m} 3$ \\
$C_{2}$ & $a r^{2} / 2, b r^{2} \cos (2 \theta) / 2, c r^{2} \sin (2 \theta) / 2$ & $\mathrm{~m} 5$ \\
$C_{1 v}\left(\sigma_{1}\right)$ & $a r \cos (\theta)$ & \\
$C_{1 v}\left(\delta_{1}\right)$ & $a r \cos (\theta-\pi / 4)$ & $\mathrm{m} 7$ \\
$C_{1 v}\left(\sigma_{2}\right)$ & $\operatorname{ar} \sin (\theta)$ & $\mathrm{m} 6$ \\
$C_{1 v}\left(\delta_{2}\right)$ & $\operatorname{ar} \sin (\theta-\pi / 4)$ & $\mathrm{m} 8$ \\
$C_{1}$ & $\operatorname{ar} \cos (\theta), b r \sin (\theta)$ & \\
\hline
\end{tabular}

Each coefficient has a size $\sim \epsilon$
If $A \neq 0$, this is a pitchfork bifurcation with a central branch $u=0$ and eigenvalue $\lambda \equiv V_{\text {,uu }}=-\mu$, and which maintains $C_{4 v}$ symmetry. From $V_{, u}=0$, the bifurcating branch gives solutions $\mu=A u^{2} / 6$ with eigenvalue $\lambda=A u^{2} / 3$, and showing $C_{2 v}(\sigma), C_{2 v}(\delta)$, and $C_{4}$ symmetry, respectively. Tables 1 and 2 show which mesh symmetries break this bifurcation, by introducing a term $a u$ in the potential from Eq. (12). Results are summarized in Table 5. When the bifurcation is broken, a limit solution will appear at $\mu \sim$ $\epsilon^{2 / 3}, u \sim \epsilon^{1 / 3}$, and when not broken, the bifurcation is displaced by $\mu \sim \epsilon$. The schematic situation is shown in Fig. $4 \mathrm{~b}$. The effects from perturbations are shown by Fig. 5c, d.

\subsection{Limit solutions of type $V$ in a conservative system}

For an equilibrium solution with $C_{4 v}$ symmetry, which has a single zero eigenvalue of type $\mathbf{V}$, the potential will to third order be

$V(u, \mu)=-\mu u+A u^{3} / 6$

with non-zero $A$, and the limit solution remains for all mesh symmetries, just being displaced by $\mu \sim \epsilon, u \sim \epsilon$. This represents the commonly known fact that limit solutions are only mildly imperfection sensitive, when compared to the different classes of structural bifurcation situations.

\subsection{Example: type I bifurcation broken by a $C_{2 v}(\sigma)$ perturbation}

As noted above, $C_{2}$ and $C_{2 v}$ perturbations of the symmetry will break the double bifurcation existing for the full symmetry $C_{4 v}$. A further analysis of these cases shows what can be the expected disturbances to the full unfolding of the double bifurcation. For a $C_{2 v}(\sigma)$ perturbation, the perturbed potential is to fourth order, Table 4 ,

$$
\begin{aligned}
V(r, \theta, \mu) & =(a-\mu) r^{2} / 2+r^{4}(A+B \cos (4 \theta)) / 24+ \\
& +b r^{2} \cos (2 \theta) / 2,
\end{aligned}
$$

assuming $B \neq 0,|A| \neq|B|$, and $b \neq 0$. Equilibrium solutions are given by

$$
\begin{aligned}
V_{, u}= & u\left[(a-\mu)\left(u^{2}+v^{2}\right)+\frac{A}{6}\left(u^{2}+v^{2}\right)\right. \\
& \left.+\frac{B}{6}\left(u^{2}-3 v^{2}\right)+b\right]=0 \\
V_{, v}= & v\left[(a-\mu)\left(u^{2}+v^{2}\right)+\frac{A}{6}\left(u^{2}+v^{2}\right)\right. \\
& \left.+\frac{B}{6}\left(v^{2}-3 u^{2}\right)-b\right]=0 .
\end{aligned}
$$

The solution branches, and the resulting bifurcations corresponding to these local equilibrium equations are given in Appendix 1.

\subsection{Example: type I bifurcation broken by $C_{1 v}\left(\sigma_{1}\right)$ perturbation}

Perturbations of type $C_{1 v}$ and $C_{1}$ will replace even the central branch. Similarly as above, the perturbed potential for a $C_{1 v}\left(\sigma_{1}\right)$ perturbation, is to fourth order

$$
\begin{aligned}
V(r, \theta, \mu) & =-\mu r^{2} / 2+r^{4}(A+B \cos (4 \theta)) / 24+ \\
& +a r \cos (\theta),
\end{aligned}
$$

assuming $B \neq 0,|A| \neq|B|$, and $a \neq 0$. Equilibrium solutions are then given by 


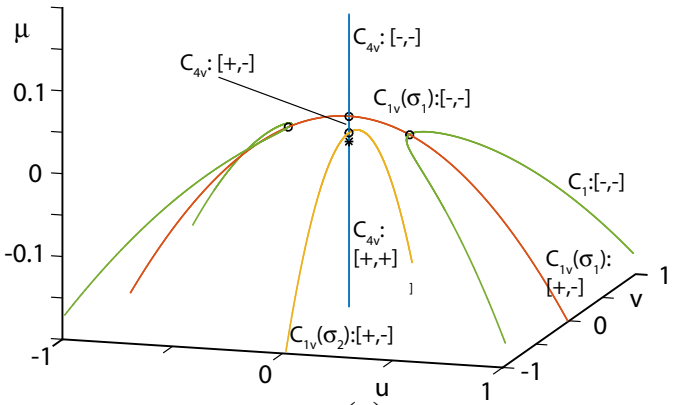

(a)

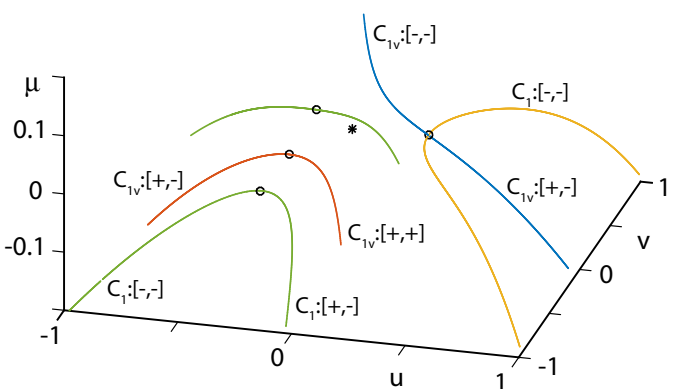

(b)

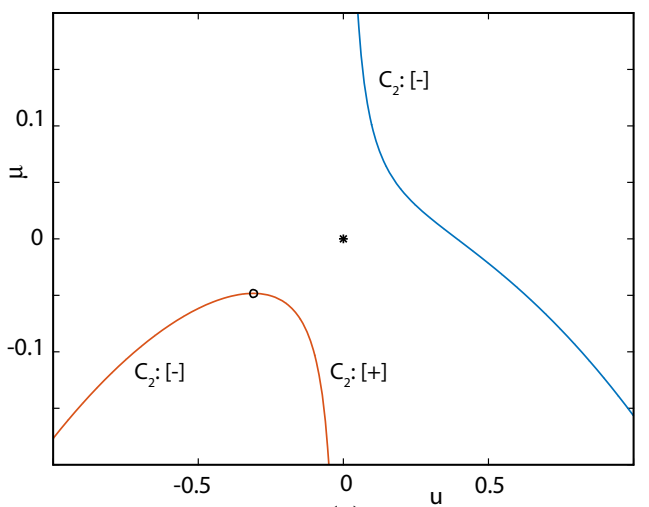

(c)

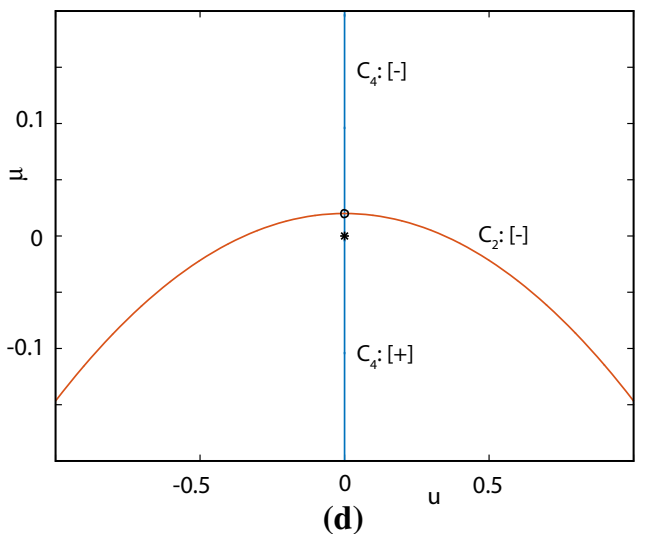

Fig. 5 Example of bifurcations affected by perturbations. $\mathbf{a}-\mathbf{b}$ : type $\mathbf{I}$ with $A=-1, B=-0.4$ broken by $C_{2 v}(\sigma)(a=0.02, b=0.01)$, and $C_{1 v}\left(\sigma_{1}\right)(a=0.01)$ perturbations, respectively. $\mathbf{c}-\mathbf{d}$ : type II with $A=-1$ broken by $C_{2}(a=0.01)$, and $C_{4}(a=0.02)$, respectively. The star denotes the unperturbed situation, the rings bifurcations or limit solutions on the branches. Labels on curves show the symmetry of the solutions, and the signs of the relevant eigenvalues

$$
\begin{aligned}
V_{, u}= & -\mu u\left(u^{2}+v^{2}\right)+\frac{A}{6} u\left(u^{2}+v^{2}\right)+ \\
& +\frac{B}{6} u\left(u^{2}-3 v^{2}\right)+a=0 \\
V_{, v}= & -\mu v\left(u^{2}+v^{2}\right)+\frac{A}{6} v\left(u^{2}+v^{2}\right)+ \\
& +\frac{B}{6} v\left(v^{2}-3 u^{2}\right)=0 .
\end{aligned}
$$

The first equation shows that $u \neq 0$ at any equilibrium solution. The $\mu$ dependence can be isolated by re-writing these equations as

$$
\begin{aligned}
& u V_{, v}-v V_{, u}=V_{, \theta} \\
&=- v\left(\frac{2 B}{3}\left(u^{3}-u v^{2}\right)+a\right)=0 \\
& u V_{, u}+v V_{, v}=r V_{, r} \\
&=-\mu\left(u^{2}+v^{2}\right)+\frac{A}{6}\left(u^{2}+v^{2}\right)^{2}+ \\
& \quad+\frac{B}{6}\left(u^{4}-6 u^{2} v^{2}+v^{4}\right)+a u=0,
\end{aligned}
$$

Equation (19) gives solutions with either $v=0$ (branches with $C_{1 v}\left(\sigma_{1}\right)$ symmetry) or $v^{2}=u^{2}+3 a /(2 B u)$ (branches with $C_{1}$ symmetry), and then $\mu$ can be solved from Eq. (20). These solutions characterize the solution branches and critical solutions which will appear for a perturbed element mesh. The full catalogue of analytical results is given in Appendix 2 .

\section{Numerical modelling}

We studied a thin, horizontal square membrane of unstretched measures $40 \times 40 \mathrm{~mm}^{2}$ and thickness $D_{0}=$ $0.01 \mathrm{~mm}$, uniformly pre-stretched to $160 \times 160 \mathrm{~mm}^{2}$, and affected by a hydro-static pressure from below. The MooneyRivlin model, Eqs. (1-2), used $\mu=0.4225 \mathrm{MPa}, k=0.1$. Loading came from a fluid of density $\rho=1 \cdot 10^{-6} \mathrm{~kg} / \mathrm{mm}^{3} \approx$ $\rho_{\mathrm{H}_{2} \mathrm{O}}$, and a gravity acceleration of $g=9.81 \mathrm{~m} / \mathrm{s}^{2}$. Fluid level $Z_{\text {fluid }}$, measured from the initial horizontal membrane plane was the primary load parameter, but the fluid volume $V_{\text {fluid }}$ enclosed by the membrane was seen as a secondary parameter [44].

\subsection{Critical equilibrium solutions on fundamental branch}

A solution was calculated through a mesh m1(32768), Fig. 2. The fundamental branch with mid-point spatial coordinates $\left(x_{m}, y_{m}\right)=(0,0)$ is expressed as the midpoint spatial coordinate $z_{m}$ in Fig. 6, with fluid level and fluid volume as load parameters. A set of critical equilibrium solutions were accurately isolated: a double bifurcation, two single bifurcations, 
Table 5 Mesh symmetries that break the pitchfork bifurcations of types II-IV, and mesh classes in Fig. 2 which will thereby hide the bifurcation

\begin{tabular}{lll}
\hline Type & Mesh symmetries that break the bifurcation & Mesh classes \\
\hline II & $C_{2 v}(\sigma), C_{2}, C_{1 v}\left(\sigma_{1}\right), C_{1 v}\left(\sigma_{2}\right), C_{1}$ & $\mathrm{~m} 2, \mathrm{~m} 5, \mathrm{~m} 6, \mathrm{~m} 8$ \\
III & $C_{2 v}(\delta), C_{2}, C_{1 v}\left(\delta_{1}\right), C_{1 v}\left(\delta_{2}\right), C_{1}$ & $\mathrm{~m} 3, \mathrm{~m} 5, \mathrm{~m} 7, \mathrm{~m} 8$ \\
IV & $C_{4}, C_{2}, C_{1}$ & $\mathrm{~m} 4, \mathrm{~m} 5, \mathrm{~m} 8$ \\
\hline
\end{tabular}

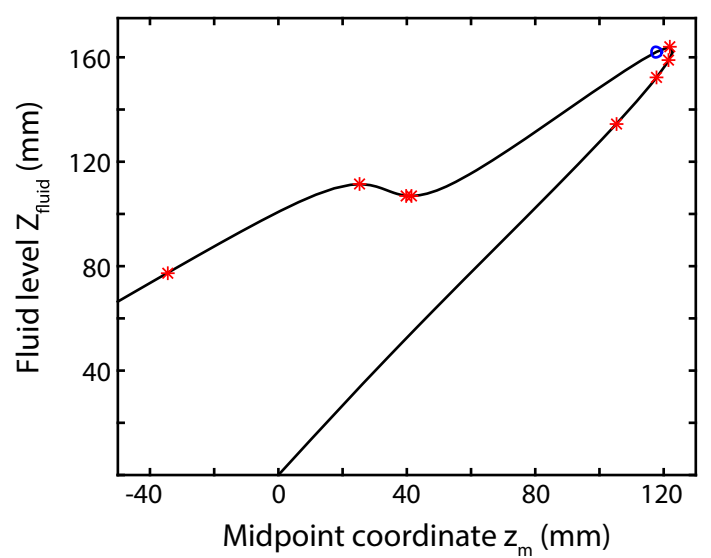

(a)

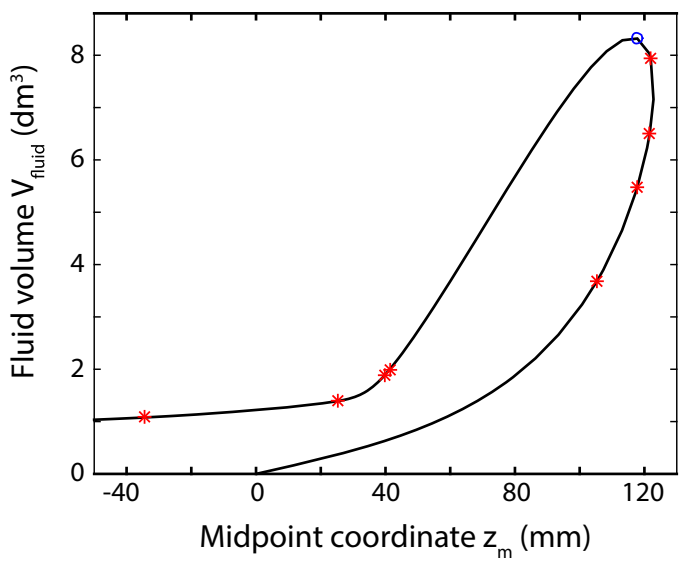

(b)

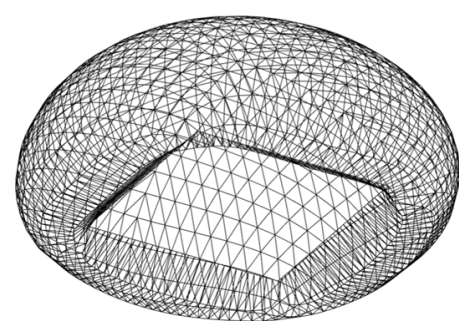

(c)

Fig. 6 Square membrane analysed with a mesh m1(32768), Fig. 2. Fundamental branch. Midpoint spatial coordinate $z_{m}$ as function of fluid level $Z_{\text {fluid }}$ in a, and of enclosed fluid volume $V_{\text {fluid }}$ in $\mathbf{b}$. Curves are discontinued at the solution where wrinkling first appeared. Stars and circles indicate bifurcation and limit solutions. Subfigure $\mathbf{c}$ shows deflected shape at the maximum fluid level, projected on the m1(2048) mesh

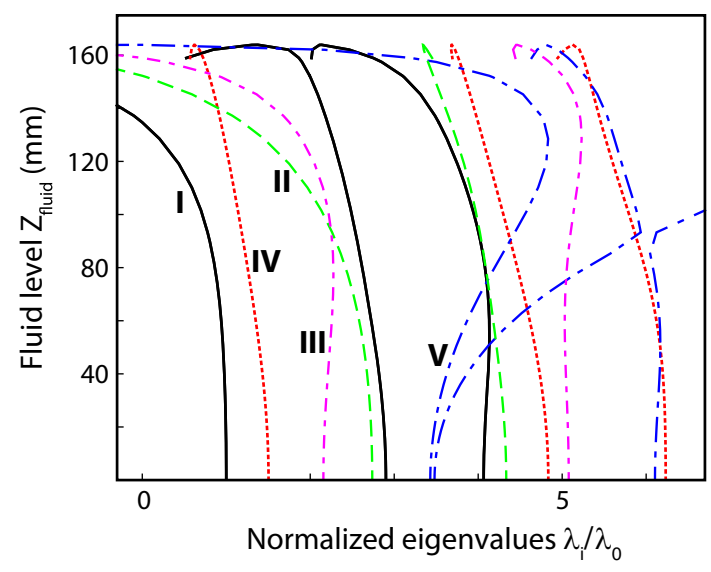

Fig. 7 Selection of the lowest eigenvalues of each type for mesh $\mathrm{m} 1(32768)$ as functions of fluid level $Z_{\text {fluid. }}$. Eigenvalues normalized to the lowest eigenvalue at the first non-zero equilibrium solution on the fundamental branch. Eigensolution types are marked on one curve of each type

two limit solutions in fluid level, a single bifurcation, a limit solution and a double bifurcation. The limit solution with respect to fluid volume was isolated from the changing sign of its component in the tangent vector.

The critical solutions up to the maximum fluid level can be seen in Fig. 7, from the zero-crossings of a set of eigenvalues of the tangential stiffness matrix on the fundamental branch. The eigensolutions are classified into the types $\mathbf{I}-\mathbf{V}$ described in 3.3. It is noted how eigenmodes from different types will be independent and how their eigenvalues can cross when the loading parameter is varied. This means that the eigenvalues will coincide and give a two-dimensional eigenspace where the component modes will belong to different symmetry groups. Eigenmodes within the same type, however, typically show a mode interaction in the form commonly known as 'mode veering' [14,25], where two modes approach each other, exchange mode shapes and diverge again during a parameter variation. A second parameter in the problem is needed to create the two-dimensional eigenspace for the common eigenvalue.

Table 6 shows the fluid levels $Z_{\text {fluid }}$ for the critical solutions for a set of meshes of type $\mathrm{m} 1$. These are compared to a set of solutions obtained by very fine discretizations of subdomain ' $\mathrm{gO}$ ' with high-order elements, and the different boundary conditions from Table 3. These solutions are believed to be fully converged to the continuous case. It is 
Table 6 Critical fluid levels for element meshes of type $\mathrm{m} 1$, Fig. 2

\begin{tabular}{lllllll}
\hline Mesh & 'Converged' & $\mathrm{m} 1(32768)$ & $\mathrm{m} 1(8192)$ & $\mathrm{m} 1(2048)$ & $\mathrm{m} 1(512)$ & $\mathrm{m} 1(128)$ \\
\hline bif. 1 (I) & 134.5749 & 134.69 & 135.02 & 136.31 & 141.20 & 158.84 \\
bif. 2 (II) & 151.9953 & 152.25 & 153.00 & 155.95 & 167.93 & 231.24 \\
bif. 3 (III) & 158.5104 & 158.98 & 160.29 & 165.09 & 183.17 & 270.23 \\
lim. (V) & 163.2871 & 163.90 & 165.55 & 171.49 & 195.08 & 357.18 \\
lim. (V) & & 106.91 & 107.70 & $110.70^{*}$ & 115.68 & - \\
bif. 4 (I) & & 107.00 & 107.79 & $110.73^{*}$ & 115.88 & - \\
lim. (V) & & 111.40 & 109.72 & $110.81^{*}$ & 115.96 & - \\
bif. 5 (I) & & 77.40 & - & - & - & - \\
\hline
\end{tabular}

Notation '-' indicates that the critical solution was not found or that other response phenomena disguised the visibility, and ' $*$ ' that the solution was reached after partial inversion of the model, including local critical solutions along this process. Roman numbers within parentheses indicate the type of critical eigensolution, cf. 3.3 noted that the first four critical solutions will be found in the right order with even very coarse meshes, with an error in critical fluid levels approximately proportional to $h^{2}$, with $h$ the representative element size. Beyond the maximum fluid level solution, some critical solutions will be re-ordered or disappearing for coarse meshes, due to extensive local deformations.

\subsection{Simulations on subdomain meshes}

Simulations were also performed with a mesh $\mathrm{mH}(4096)$, being one half of mesh m1(8192), and utilizing the most obvious mirror symmetry $C_{1 v}\left(\sigma_{1}\right)$, symbolically described as $y(X, 0)=0$, cf. 3.2, for both solution and eigenmodes. This model exactly reproduced the fundamental solution, and showed the critical fluid levels for case m1(8192) in Table 6, except 'bif. 3'. Further, the double critical solutions 'bif. 1' and 'bif. 4' were indicated as single. As discussed in 3.3 and 3.4 , the eigenmodes fulfilling this symmetry are $u \phi_{1}$ for type $\mathbf{I}$, and $u \phi$ for types II and $\mathbf{V}$, but no others.

The same mesh was used also with other boundary conditions, using the $r_{2}$ element of the symmetry group $C_{2}$, and introducing boundary conditions as:

$\langle x, y, z\rangle(X, 0)=\langle-x,-y, z\rangle(-X, 0)$.

Solving the problem with this mesh and these boundary conditions gave the same fundamental solution, but missed 'bif. 1 ' and 'bif. 4' of type $\mathbf{I}$.

It is noted from these results that either set of boundary conditions gave the fundamental solution branch, and also found the limit point. Different bifurcations, and corresponding secondary solution branches were, however, found. Neither of the solutions found the full description of 'bif. 1', since at least one secondary branch did not fulfill the symmetry conditions. In order to obtain the critical directions, the solution of the equilibrium problem must be supplemented with an eigenanalysis of a problem where other boundary conditions are introduced. A complete description of the instability response must thereby analyse the full tangential stiffness matrix, but condensed in several ways, reflecting all the group elements needed to make the model complete.

The quarter mesh $\mathrm{mQ}(2048)$ with $s_{0}$ and $s_{2}$ symmetries, and introducing the mirror kinematic constraints $y(X, 0)=0$ and $x(0, Y)=0$, i.e., $C_{2 v}(\sigma)$ symmetry for both solution and eigenmodes, reproduced only the limit solutions and 'bif. 2'. With the boundary conditions given as

$\langle x, y, z\rangle(0, Y)=\langle-y, x, z\rangle(Y, 0)$,

i.e., $C_{4}$ symmetry, only the limit solutions were found, as no instability of type IV is present in the problem. Similarly, the mesh mD(4096), with $C_{1 v}\left(\delta_{1}\right)$ symmetry, missed 'bif. 2', and showed only a simple bifurcation at 'bif. 1 ', corresponding to the mode $u\left(\phi_{1}+\phi_{2}\right)$, whereas the mesh mT(2048), with $C_{2 v}(\delta)$ symmetry, missed both 'bif. 1' and 'bif. 2'. Otherwise, results agreed exactly with those for $\mathrm{m} 1$ (8192) given in Table 6 . The results verify that the statements in 3.3 are valid for the discretizations of subdomains of the whole square, and emphasize that several sets of different boundary conditions are needed in order to obtain all critical solutions, when using a subdomain.

\subsection{Meshes with lower symmetry}

When analyzing the full square with meshes of lower symmetry, other effects on the critical solutions were obtained. The simulations focussed on moderately refined discretizations. Due to the above noted local deformations when progressing far on the equilibrium branch-highly mesh-dependent in their details - only the four first critical solutions from Table 6 are shown in Table 7. The different meshes split the double bifurcation into two single ones, or break the bifurcation into limit solutions according to the statements in 3.6. 
Table 7 Critical fluid levels corresponding to the first four critical solutions in Table 6 obtained with different element meshes, Fig. 2

\begin{tabular}{llllllll}
\hline Mesh & $\mathrm{m} 2(6144)$ & $\mathrm{m} 3(3072)$ & $\mathrm{m} 4(4096)$ & $\mathrm{m} 5(3584)$ & $\mathrm{m} 6(2560)$ & $\mathrm{m} 7(2560)$ & $\mathrm{m} 8(2304)$ \\
\hline bif. 1 & 135.01 & 135.42 & 136.55 & 135.12 & $134.62(\mathrm{~L})$ & 133.93 & $134.63(\mathrm{~L})$ \\
& 135.06 & 135.87 & & 135.96 & 136.54 & $133.95(\mathrm{~L})$ & \\
bif. 2 & $152.75(\mathrm{~L})$ & 154.32 & 156.69 & $151.52(\mathrm{~L})$ & $155.49(\mathrm{~L})$ & 157.60 & $155.34(\mathrm{~L})$ \\
bif. 3 & 160.13 & $159.96(\mathrm{~L})$ & 161.63 & $159.26(\mathrm{~L})$ & 164.39 & $162.46(\mathrm{~L})$ & $163.86(\mathrm{~L})$ \\
lim. & 165.39 & 168.30 & 168.30 & 168.50 & 169.99 & 171.00 & 169.88 \\
\hline
\end{tabular}

Double values indicate that the double critical solution was split into two, and (L) that the bifurcation was replaced by a limit solution

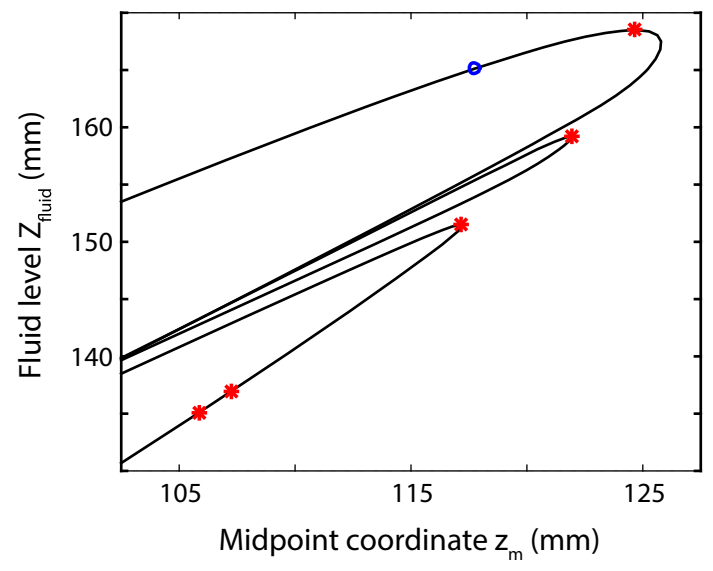

(a)

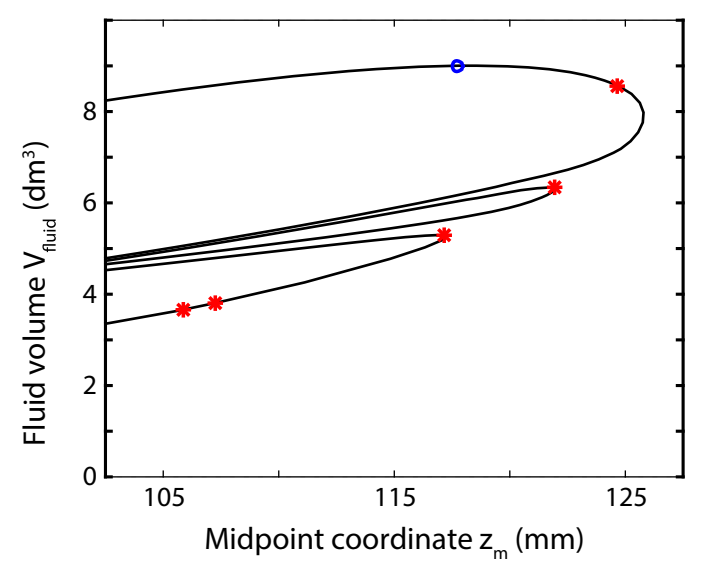

(b)

Fig. 8 Extract from fundamental solution branches for mesh $\mathrm{m5}(3584)$, around the first critical solutions. Midpoint spatial coordinate $z_{m}$ as function of fluid level $Z_{\text {fluid }}$ in a, and of fluid volume $V_{\text {fluid }}$ in b. Stars are solutions with vanishing eigenvalues, ring is the isolated solution of maximum fluid volume

The fundamental branch figure obtained for the mesh m5(3584) of symmetry $C_{2}$ is shown in Fig. 8. The type $\mathbf{I}$ bifurcation is split into two single bifurcations, whereas the types II and III are broken into limit solutions from this type of mesh perturbation, which only respects the two-fold rotation symmetry $C_{2}$, agreeing with Table 5 and Fig. 5 d.
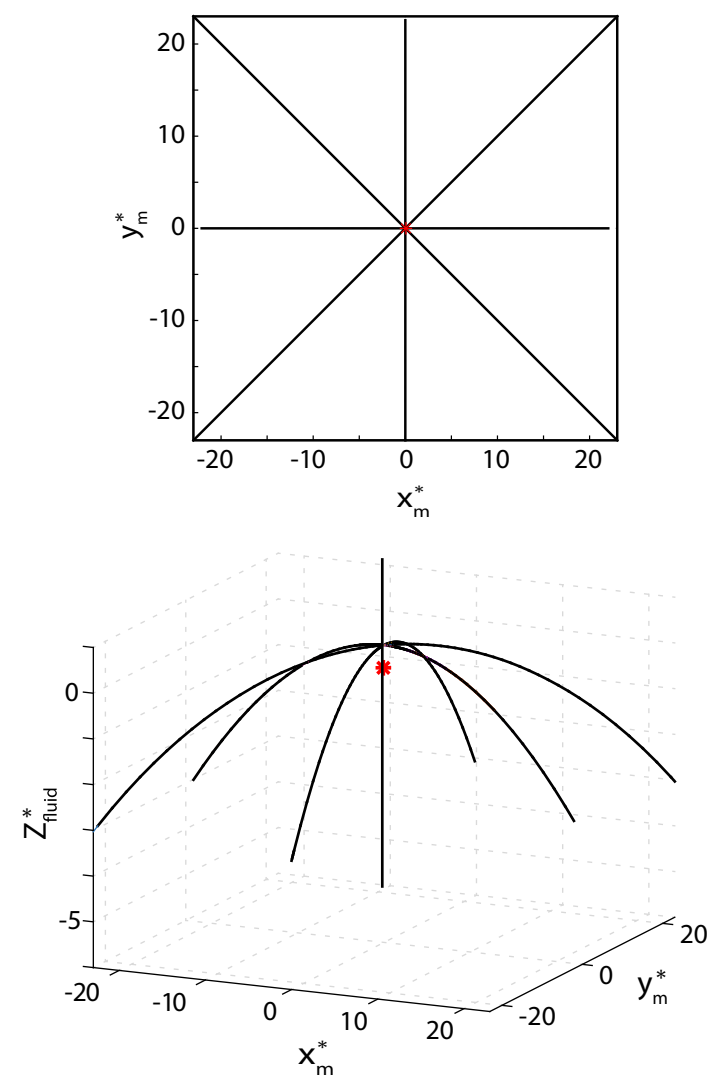

Fig. 9 Mesh m1(8192). Solution branches around 'bif. 1', represented by mid-point spatial coordinate differences $\left(x_{m}^{*}, y_{m}^{*}, z_{m}^{*}\right)$, and fluid level $Z_{\text {fluid }}^{*}$, Eq. (21)

The convergence of the limit solutions corresponding to the broken first bifurcation with mesh fineness is shown below.

\subsection{Secondary branches for $C_{4 v}$ meshes $\mathrm{m} 1$}

The symmetry properties of meshes for the whole domain affect the secondary branches emanating at the bifurcations. These were studied, starting from the m1(32768) model. At the first, double bifurcation, a two-dimensional critical eigenspace exists, which is well described by the mid-point spatial coordinates $\left(x_{m}, y_{m}\right)$. Four secondary equilibrium branches 


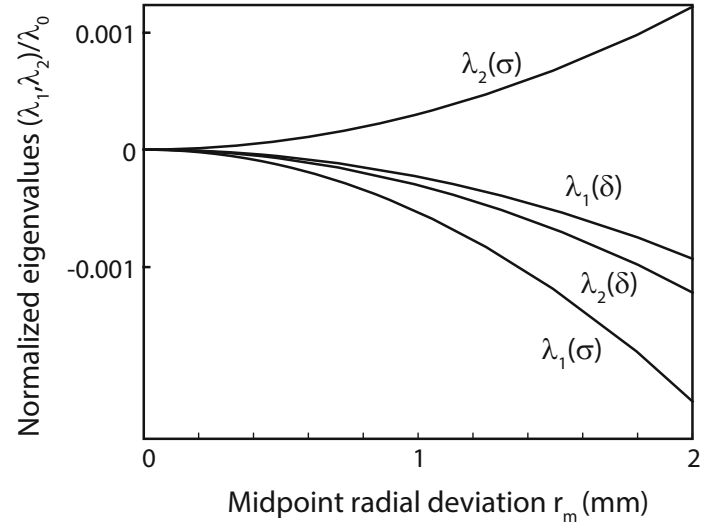

(a)

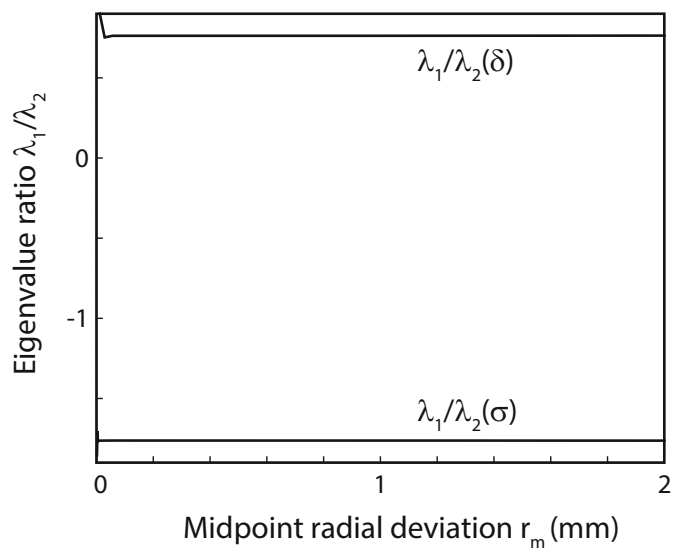

(b)

Fig. 10 Eigenvalue variations for mesh m1(328768) on secondary paths emanating from 'bif. 1', as functions of in-plane midpoint spatial radial position. Subfigure a shows normalized eigenvalues, $\mathbf{b}$ the ratios $\lambda_{1} / \lambda_{2}$, with definition in 3.5

from the bifurcation were found. A pictorial description is that the inflated bubble falls down over either one edge, or over one corner, keeping one mirror symmetry. All secondary branches were found unstable with respect to the primary load parameter $Z_{\text {fluid }}$, leading to a sinking fluid level with increasing radius, and one or two negative eigenvalues in the tangential stiffness matrix.

The single bifurcations 'bif. 2' and 'bif. 3' gave secondary branches with $x_{m}=y_{m}=0$, and were found unstable, whereas the double bifurcation 'bif. 4' gave stable secondary branches. These will not be further discussed in this study.

For discussion of the secondary equilibrium solutions emanating at the double bifurcation 'bif. 1', the difference from the converged bifurcation is introduced:

$$
\begin{aligned}
& x_{m}^{*}=x_{m}-0 ; \quad y_{m}^{*}=y_{m}-0 ; \\
& z_{m}^{*}=z_{m}-105.3112 \\
& Z_{\text {fluid }}^{*}=Z_{\text {fluid }}-134.5749
\end{aligned}
$$
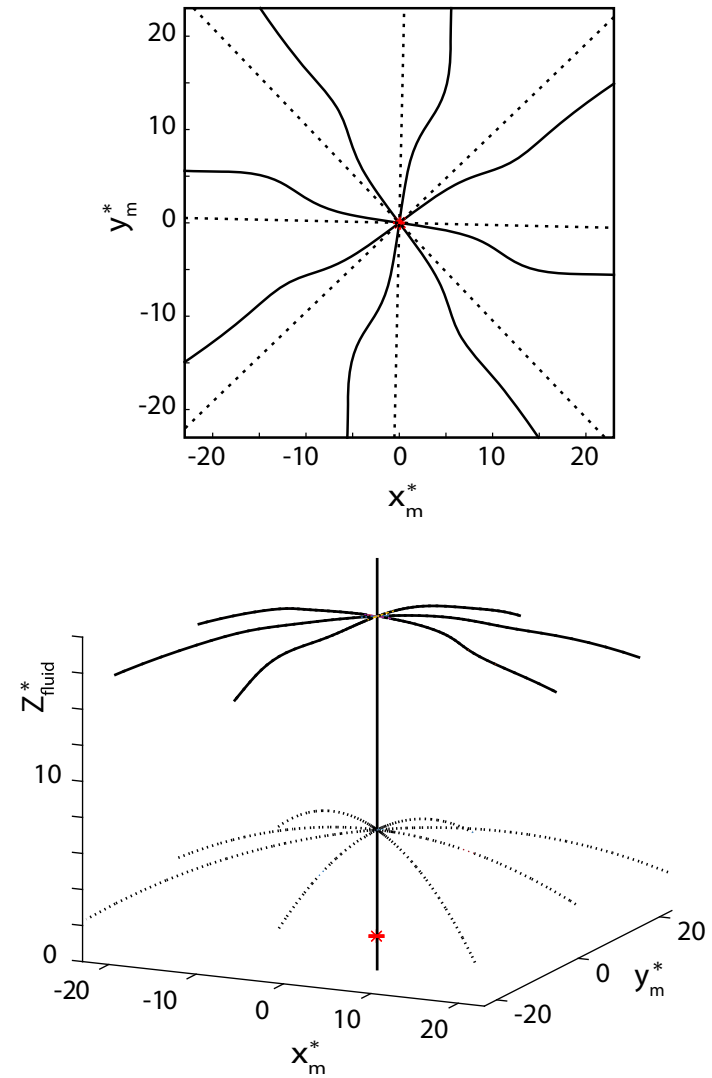

Fig. 11 Solution branches around 'bif. 1' for meshes m4(256) (solid) and $\mathrm{m} 4(1024)$ (dashed), represented by mid-point spatial coordinate differences $\left(x_{m}^{*}, y_{m}^{*}, z_{m}^{*}\right)$, and fluid level $Z_{\text {fluid }}^{*}$, Eq. (21)

For meshes of type $\mathrm{m} 1\left(C_{4 v}\right)$, the secondary response branches are characterized as, respectively, $x_{m}=0, y_{m}=$ $0, y_{m}=x_{m}$ and $y_{m}=-x_{m}$, illustrated for the mesh m1(8192) by two projections in Fig. 9, cf. Fig. 4a. The secondary branches were discontinued when first wrinkling was noted.

The lowest two eigenvalues along the secondary branches emanating from 'bif. 1' were evaluated for the mesh $\mathrm{m1}$ (32768), and varied according to Fig. 10a as functions of the midpoint spatial coordinate $r_{m}=\left(x_{m}^{2}+y_{m}^{2}\right)^{1 / 2}$ for secondary paths of type $\delta_{1}$ and $\sigma_{1}$, respectively. Both the eigenvalues vanishing at 'bif. 1' become negative on a secondary path of type $\delta$, whereas one becomes positive for a path of type $\sigma$. Figure 10b shows the obtained variation of the ratios $\lambda_{1} / \lambda_{2}$ for these paths, with the definition of the two eigenvalues from 3.5 . At $r_{m}=0.13 \mathrm{~mm}$, the ratios were evaluated as -1.7624 (for the $\sigma$ case), and +0.7624 (for the $\delta$ case). Using Eqs. (11), these values were used to evaluate the constant ratio $B / A=0.396$. From similar evaluations for the $\mathrm{m} 1(2048) \mathrm{mesh}$, the two $\lambda_{1} / \lambda_{2}$ ratios were obtained as -1.7705 and +0.7705 , respectively, yielding the ratio $B / A=0.394$. It is noted that the sum of the two ratios is -1 , independent of the mesh fineness. 

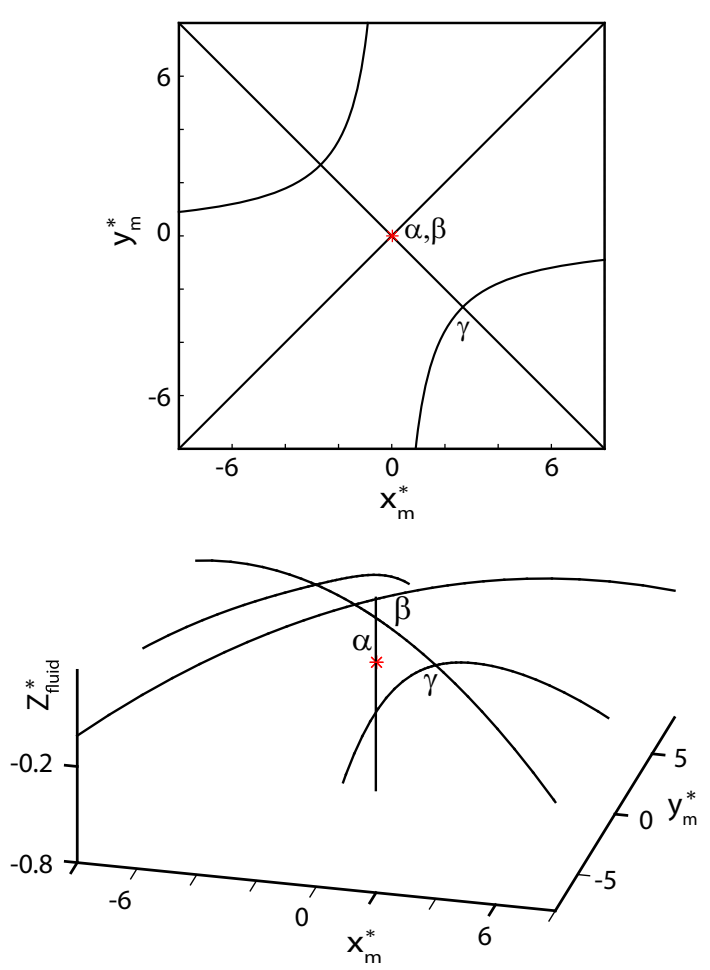

Fig. 12 Solution branches around 'bif. 1' for mesh m3(12288), represented by mid-point spatial coordinate differences $\left(x_{m}^{*}, y_{m}^{*}, z_{m}^{*}\right)$, and fluid level $Z_{\text {fluid }}^{*}$, Eq. (21). Data for indicated solutions are given in Table 8

\subsection{Secondary branches for unsymmetric meshes}

The above section showed the secondary equilibrium solutions for the $C_{4 v}$ meshes of type $\mathrm{m} 1$. The results obtained for meshes of lower symmetry will be reported next.

\subsection{1 $C_{4}$ meshes $m 4$}

The meshes of type $\mathrm{m} 4$ showed a double critical solution at the first bifurcation, Table 7 , and four secondary branches. The four-fold rotation but no mirror symmetries changed the initial directions of the secondary branches.
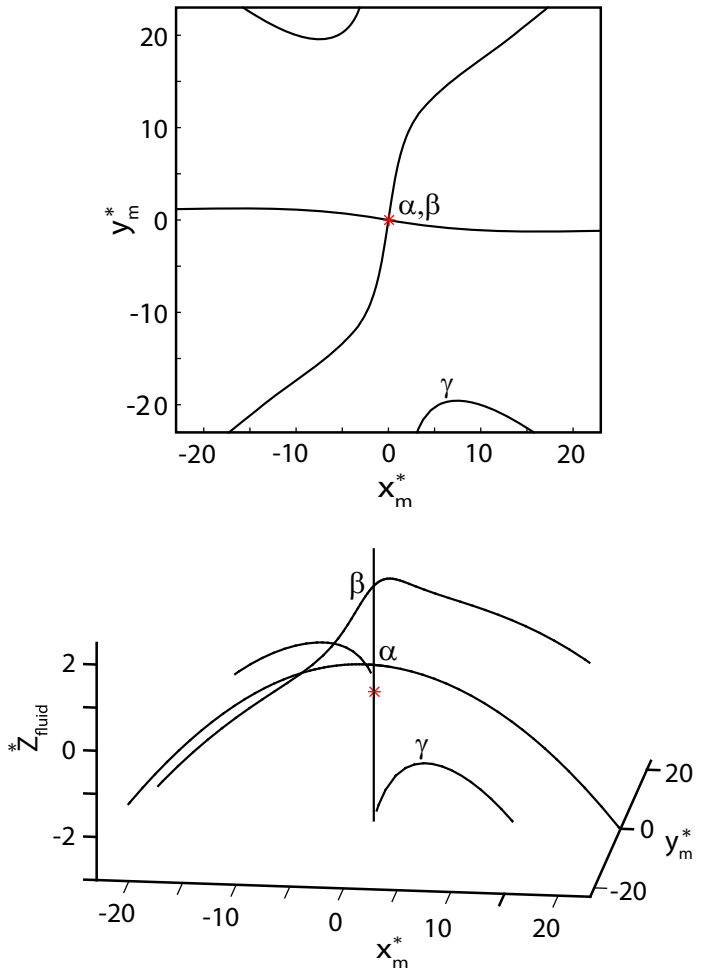

Fig. 13 Solution branches around 'bif. 1' for mesh m5(3584), represented by mid-point spatial coordinate differences $\left(x_{m}^{*}, y_{m}^{*}, z_{m}^{*}\right)$, and fluid level $Z_{\text {fluid }}^{*}$, Eq. (21). Data for indicated solutions are given in Table 9

Measured in the mid-point coordinates $\left(x_{m}^{*}, y_{m}^{*}\right)$, the outgoing branches are rotated compared to the $\mathrm{m} 1$ results, Fig. 11(left), by the same angle. This is evaluated as $\tan ^{-1}(0.146), \tan ^{-1}(0.023), \tan ^{-1}(0.0058)$ and $\tan ^{-1}$ (0.0014) for the meshes $\mathrm{m} 4(256), \mathrm{m} 4(1024), \mathrm{m} 4(4096)$ and $\mathrm{m} 4(16384)$, respectively, indicating an $h^{2}$ convergence to zero.

\subsection{2 $C_{2 v}\left(\delta_{1}\right)$ meshes $m 3$}

The $\mathrm{m} 3$ meshes split the double critical solution into two single bifurcations, Table 7. Each bifurcation gave one crossing
Table 8 Positions of bifurcation points for $\mathrm{m} 3$ meshes, cf. Fig. 12

\begin{tabular}{llllll}
\hline Solution & Variable & $\mathrm{m} 3(49152)$ & $\mathrm{m} 3(12288)$ & $\mathrm{m} 3(3072)$ & $\mathrm{m} 3(768)$ \\
\hline$\alpha:$ & $x_{m}^{*}=y_{m}^{*}$ & 0 & 0 & 0 & 0 \\
& $z_{m}^{*}$ & 0.036 & 0.148 & 0.601 & 1.784 \\
$\beta:$ & $Z_{\text {fluid }}^{*}$ & 0.054 & 0.215 & 0.844 & 3.251 \\
& $x_{m}^{*}=y_{m}^{*}$ & 0 & 0 & 0 & 0 \\
& $z_{m}^{*}$ & 0.059 & 0.236 & 0.935 & 3.610 \\
{$[2 \mathrm{~mm}] \gamma:$} & $Z_{\text {fluid }}^{*}$ & 0.084 & 0.331 & 1.291 & 4.900 \\
& $x_{m}^{*}=-y_{m}^{*}$ & 1.360 & 2.671 & 5.273 & 10.486 \\
& $z_{m}^{*}$ & 0.022 & 0.096 & 0.396 & 1.579 \\
& $Z_{\text {fluid }}^{*}$ & 0.042 & 0.171 & 0.671 & 2.575 \\
\hline
\end{tabular}


Table 9 Positions of bifurcation points for m5 meshes, cf. Fig. 13

\begin{tabular}{lllccc}
\hline Solution & Variable & $\mathrm{m} 5(57344)$ & $\mathrm{m} 5(14336)$ & $\mathrm{m} 5(3584)$ & $\mathrm{m} 5(896)$ \\
\hline$\alpha:$ & $x_{m}^{*}=y_{m}^{*}$ & 0 & 0 & 0 & 0 \\
& $z_{m}^{*}$ & 0.033 & 0.134 & 0.570 & 2.358 \\
& $Z_{\text {fluid }}^{*}$ & 0.028 & 0.121 & 0.549 & 2.393 \\
$\beta:$ & $x_{m}^{*}=y_{m}^{*}$ & 0 & 0 & 0 & 0 \\
& $z_{m}^{*}$ & 0.163 & 0.578 & 1.939 & 5.871 \\
& $Z_{\text {fluid }}^{*}$ & 0.202 & 0.729 & 2.381 & 7.103 \\
$\gamma:$ & $x_{m}^{*}$ & 1.915 & 3.741 & 7.207 & 13.045 \\
& $y_{m}^{*}$ & -5.516 & -10.575 & -19.573 & -36.142 \\
& $z_{m}^{*}$ & -0.050 & -0.191 & -0.687 & -2.500 \\
& $Z_{\text {fluid }}^{*}$ & -0.021 & -0.084 & -0.332 & -1.299 \\
& $\tan \left(\theta_{1}^{*}\right)$ & 0.1109 & 0.1195 & 0.1594 & 0.2136 \\
\hline
\end{tabular}
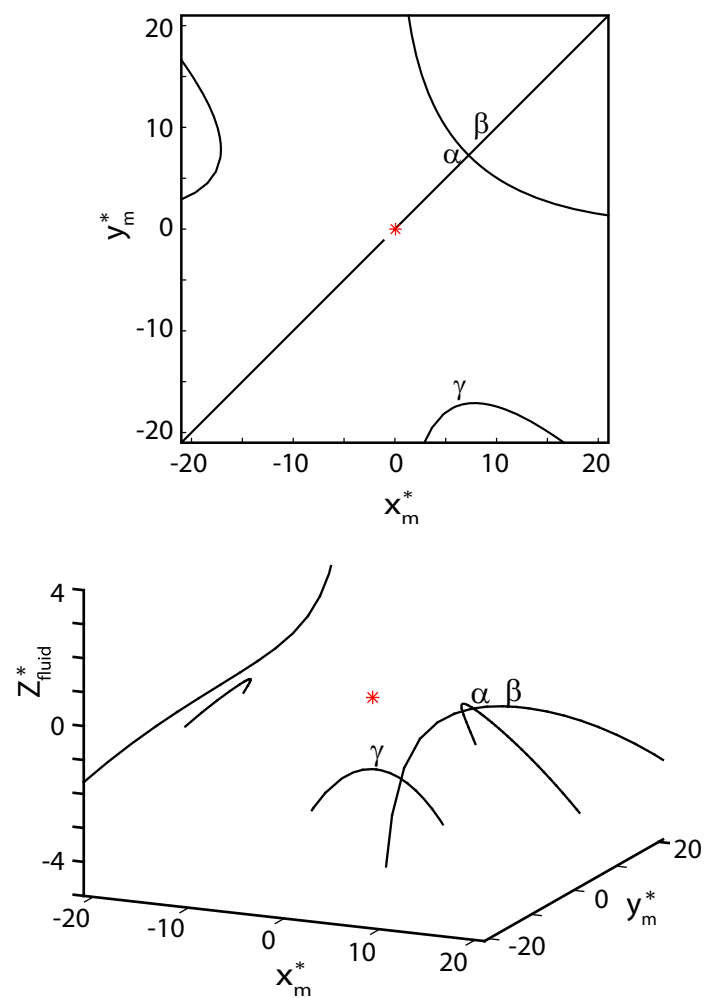

Fig. 14 Solution branches around 'bif. 1' for mesh m7(10240), represented by mid-point spatial coordinate differences $\left(x_{m}^{*}, y_{m}^{*}, z_{m}^{*}\right)$, and fluid level $Z_{\text {fluid }}^{*}$, Eq. (21). Data for indicated solutions are given in Table 10

secondary branch: the first followed $y_{m}=-x_{m}$, whereas the second followed $y_{m}=x_{m}$. The first of these also gave two symmetrically placed bifurcations, where tertiary equilibrium branches emanate, Fig. 12. The bifurcations found for some $\mathrm{m} 3$ meshes are given in Table 8 , which indicates that all $z_{m}^{*}$ and $Z_{\text {fluid }}^{*}$ converge to zero with an error proportional to $h^{2}$, while the in-plane component given converges with $h^{1}$ error, cf. Appendix 1, with $a, b \propto h^{2}$.
Table 10 Positions of bifurcation points for $\mathrm{m} 7$ meshes, cf. Fig. 14

\begin{tabular}{llrrrr}
\hline Solution & Variable & $\mathrm{m} 7(40960)$ & $\mathrm{m} 7(10240)$ & $\mathrm{m} 7(2560)$ & $\mathrm{m} 7(640)$ \\
\hline$\alpha:$ & $x_{m}^{*}=y_{m}^{*}$ & 4.912 & 7.257 & 10.449 & 14.892 \\
& $z_{m}^{*}$ & -0.394 & -0.750 & -1.133 & -0.877 \\
& $Z_{\text {fluid }}^{*}$ & -0.394 & -0.660 & -0.637 & 1.145 \\
$\beta:$ & $x_{m}^{*}=y_{m}^{*}$ & 5.463 & 8.054 & 11.237 & 15.021 \\
& $z_{m}^{*}$ & -0.405 & -0.773 & -1.169 & -0.887 \\
& $Z_{\text {fluid }}^{*}$ & -0.388 & -0.646 & -0.623 & 1.145 \\
$\gamma:$ & $x_{m}^{*}$ & 5.327 & 7.917 & 11.486 & 16.389 \\
& $y_{m}^{*}$ & -10.686 & -17.107 & -27.980 & -49.232 \\
& $z_{m}^{*}$ & -0.497 & -1.124 & -2.567 & -7.119 \\
& $Z_{\text {fluid }}^{*}$ & -0.359 & -0.697 & -1.2329 & -2.686 \\
\hline
\end{tabular}

The results for the m3(49152) mesh verify the measures for perturbations introduced by a lacking symmetry. According to 3.6, and Appendix 1, using the fluid level as the parameter $\mu$, and replacing $B$ with $-B$ in the expressions from the diagonal symmetry rather than an edge one, the constants $a=0.069 \mathrm{~mm}, b=0.015 \mathrm{~mm}$ from the solutions $\alpha$ and $\beta$, which would predict $Z_{\text {fluid }}^{*}=a+\frac{1}{2}\left(\frac{A}{B}+1\right) b=$ $0.043 \mathrm{~mm}$ value at solution $\gamma$, confirmed by the simulation result. The same calculations for the mesh m3(12288) gives $a=0.273 \mathrm{~mm}, b=0.058 \mathrm{~mm}$, indicating an $h^{2}$ dependence in the perturbation constants, and a prediction $Z_{\text {fluid }}^{*}=0.171 \mathrm{~mm}$ at $\gamma$.

It is noted that this result shows similarities, but also differences, when compared to Fig. 5a which shows the expected result for a mesh of symmetry $C_{2 v}\left(\sigma_{1}\right)$.

\subsection{3 $C_{2}\left(\delta_{1}\right)$ meshes $m 5$}

The $\mathrm{m} 5$ meshes also split the double critical solution into two simple bifurcations, Table 7. Each bifurcation showed one secondary branch, the first starting in a rotated direction in relation to the $x$ and $y$ axes, Fig. 13. The angle $\theta_{1}^{*}$ is dependent on the mesh fineness, but contrary to the $\mathrm{m} 4$ case above does not converge towards zero. The second secondary branch started close to $\sigma_{1}: x_{m}^{*}=0$, rotated an equal angle, but approaches a direction represented by $\delta_{1}: y_{m}^{*}=x_{m}^{*}$. Two detached branches also each connected two of the exits in Fig. 9. In order to find these, generalized equilibrium path evaluations were needed [8]. Three relevant solutions, marked in Fig. 13, are given in Table 9, together with the angle $\theta_{1}^{*}$; this was evaluated from the first (small) branch step on the secondary branch.

\subsection{4 $C_{1 v}\left(\delta_{1}\right)$ meshes $m 7$}

The $\mathrm{m} 7$ meshes broke the first bifurcation into a simple bifurcation and a limit solution with respect to $Z_{\text {fluid }}$, Table 7 . The 

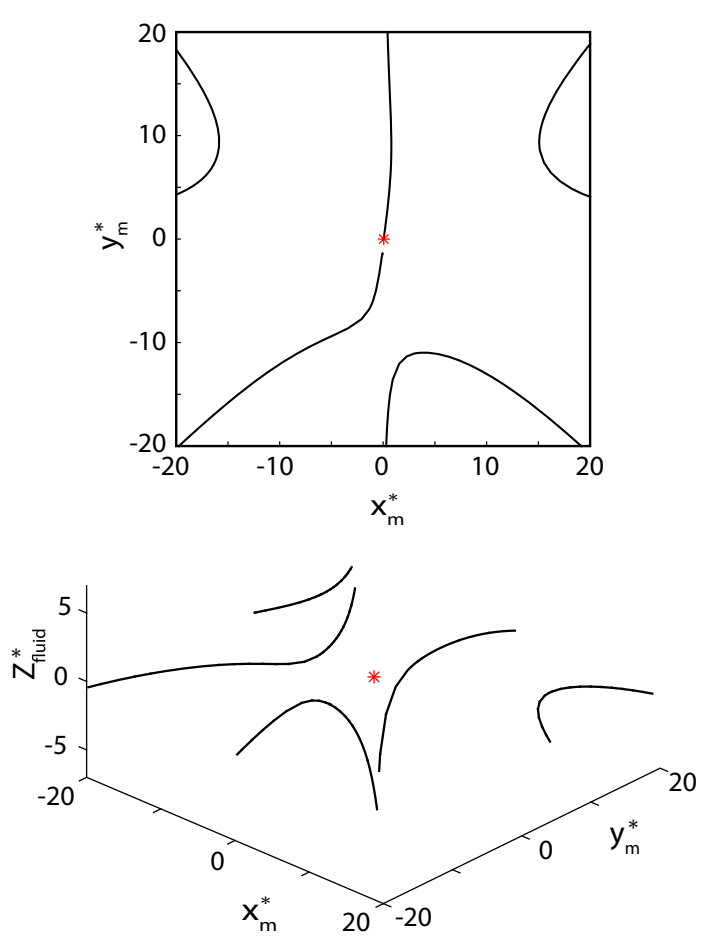

Fig. 15 Solution branches around 'bif. 1' for mesh m8(2304), represented by mid-point spatial coordinate differences $\left(x_{m}^{*}, y_{m}^{*}, z_{m}^{*}\right)$, and fluid level $Z_{\text {fluid }}^{*}$, Eq. (21)

bifurcation consistently appeared slightly before the limit solution, and showed non-zero $x_{m}=y_{m}$. Bifurcation 'bif. 1' gave a secondary branch where the mid-point initially moved in a direction $\delta x_{m}^{*}=-\delta y_{m}^{*}$, but approached the $x$ and $y$ axes. Two detached branches were found by generalized branchfollowing, Fig. 14, Table 10.

It is noted that this result shows similarities, but also differences, when compared to Fig. 5b, which shows the expected result for a mesh of symmetry $C_{1 v}\left(\sigma_{1}\right)$.

\section{6 $C_{1}$ meshes $\mathrm{m8}$}

The m8 meshes without any symmetries in the mesh, showed only limit solutions, Table 7 . The branches around the bifurcation 'bif. 1' are shown in Fig. 15.

\section{Concluding remarks}

The paper has described how symmetry aspects of a structure, and in particular of a discretization mesh on a structure, will affect the results obtained from a numerical simulation. Some aspects on the different possible symmetries in a simple geometric shape, and an analytical treatment of the responses in a square plane domain, are given as background to a set of performed simulations.

From the analytical treatment is concluded that the studied square membrane with respect to symmetry can show five different types of eigenmodes, and thereby of critical solutions. Out of these five, four are easily found for the considered problem. The instability class IV in Section 3.3, which can be seen as based on a rotation around the transversal $Z$-axis, Fig. 3, was only found in one instance: for the mesh m1(8192), and after rather severe local deformations.

The main conclusion is that the chosen discretization of a domain can significantly affect the calculated response to loading, and in particular the instability response. Using simple mirror symmetries to extract a subdomain of the whole, and introducing only the most obvious kinematic conditions on the mirror planes, can hide or distort some response aspects. Even if this approach will find the fundamental equilibrium solutions, it is necessary to consider that the eigenmodes can be situated in another solution space. This can demand the successive introduction of several different sets of boundary conditions, sometimes also solving the eigenmodes as simultaneous fields, as in Table 3. In order to find not only the eigenmodes at the critical solutions, but also secondary paths emanating from them, even more complexly combined solution fields must be sought, along the same lines as in the Table, but not further discussed here. This observation thereby supports the recommendation to use full domain modelling in problems where instabilities can be expected.

The treatment here has also discussed the symmetry and regularity of the discretization mesh for a symmetric full domain. The results show that a lacking symmetry in the mesh has a somewhat similar effect on the instability results as a distortion of the domain. The set of symmetry aspects demonstrated by the mesh types $\mathrm{m} 1-\mathrm{m} 8$ can thereby be compared to less symmetric structures, both cases representing a perturbation to the more symmetric situation. The analysis predicts which instabilities will be affected by reducing the symmetry in the problem, and these predictions are both qualitatively and quantitatively confirmed by the numerical simulations.

The paper has only discussed the symmetry aspects of a square domain. Studies can be performed also for circular domains, which show another class of symmetry, but large parts of the analysis are similar, even if somewhat simpler. The effects from modelling either only a sector of the circle with the needed boundary conditions, or the whole circular domain with a meshing based on sector subdivision, are thereby possible to evaluate, as a function of the number of sectors considered. 
Open Access This article is distributed under the terms of the Creative Commons Attribution 4.0 International License (http://creativecomm ons.org/licenses/by/4.0/), which permits unrestricted use, distribution, and reproduction in any medium, provided you give appropriate credit to the original author(s) and the source, provide a link to the Creative Commons license, and indicate if changes were made.

\section{Appendix: Analysis of type I bifurcation broken by perturbations of lower symmetry}

\section{Appendix 1: Type I bifurcation broken by a $C_{2 v}(\sigma)$ perturbation}

Equations (14-15) allow the following solutions.

Branch with $C_{2 v}(\sigma)$ symmetry: $u=0, v=0$.

Branch with $C_{1 v}\left(\sigma_{1}\right)$ symmetry: $v=0, \mu=a+(A+$ B) $u^{2} / 6+b$.

Branch with $C_{1 v}\left(\sigma_{2}\right)$ symmetry: $u=0, \mu=a+(A+$ B) $u^{2} / 6-b$.

Branches with $C_{1}$ symmetry: If $b / B>0: v^{2}=u^{2}+$ $3 b / B, \mu=a+(A-B) u^{2} / 3+(A-B) b /(2 B)$. If $b / B<$ $0: u^{2}=v^{2}-3 b / B, \mu=a+(A-B) v^{2} / 3-(A-$ $B) b /(2 B)$.

A set of bifurcations are identified, according to:

Pitchfork bifurcation from $C_{2 v}(\sigma)$ to $C_{1 v}\left(\sigma_{1}\right): u=v=$ $0, \mu=a+b$.

Pitchfork bifurcation from $C_{1 v}\left(\sigma_{1}\right)$ to $C_{1}$ : If $b / B>0$ : $v=0, u^{2}=3 b / B, \mu=a+(A-B) b /(2 B)$.

Pitchfork bifurcation from $C_{2 v}(\sigma)$ to $C_{1 v}\left(\sigma_{2}\right): u=v=$ $0, \mu=a-b$.

Pitchfork bifurcation from $C_{1 v}\left(\sigma_{2}\right)$ to $C_{1}$ : If $b / B<0$ : $u=0, v^{2}=-3 b / B, \mu=a-(A-B) b /(2 B)$.

No limit solutions exist locally.

\section{Appendix 2: Type I bifurcation broken by a $C_{1 v}\left(\sigma_{1}\right)$ perturbation}

Equations (19-20) allow solutions characterized as:

Branches with $C_{1 v}\left(\sigma_{1}\right)$ symmetry: $v=0, \mu=(A+$ $B) u^{2} / 6+a / u, u \neq 0$. These are two separate branches, for $u>0$ and $u<0$, respectively.

Branches with $C_{1}$ symmetry: $v^{2}=u^{2}+3 a /(2 B u), \mu=$ $(A-B) u^{2} / 3+(A+B) a /(4 B u)$. Allowed $u$ values are: $\operatorname{sgn}(a / B) u>0$ (two disconnected branches) or $\operatorname{sgn}(a / B) u^{3} \leq-(3 / 2)|a / B|$ (a single branch connected to a $C_{1 v}\left(\sigma_{1}\right)$ branch).
Secondary bifurcations can also be located, where a secondary $C_{1}$ branch connects with a tertiary $C_{1 v}\left(\sigma_{1}\right)$ branch. By seeking solutions where $\mu(u)$ has a local extremum, a limit solution can be located on the other $C_{1 v}\left(\sigma_{1}\right)$ branch, and possibly limit solutions on the $C_{1}$ branches. These are:

Pitchfork bifurcation: $u^{3}=-3 a /(2 B), v=0, \mu=$ $(A-3 B) u^{2} / 6, A / B \neq-3, A / B \neq 3 / 5$.

Limit solution on a $C_{1 v}\left(\sigma_{1}\right)$ branch: $u^{3}=3 a /(A+$ $B), v=0, \mu=(A+B) u^{2} / 2, A / B \neq-3$.

Limit solutions on $C_{1}$ branches: $u^{3}=3(A+B) a /(8(A-$ $B) B), v^{2}=u^{2}+3 a /(2 B u), \mu=(A-B) u^{2}$. These exists only for $A / B>1$ or $A / B<-1$ (one limit solution on each disconnected branch) or $3 / 5<A / B<1$ (two limit solutions on the connected branch).

As $A / B \rightarrow-3$, the bifurcation and limit solution on the $C_{1 v}\left(\sigma_{1}\right)$ branch joins, and as $A / B \rightarrow 3 / 5$ the bifurcation and the limit solutions on the connected $C_{1}$ branch joins. All bifurcation and limit solutions scale as $u \sim|a|^{1 / 3}, \mu \sim$ $|a|^{2 / 3}$.

\section{References}

1. Antman S, Schagerl M (2005) Slumping instabilities of elastic membranes holding liquids and gases. Int $\mathrm{J}$ Non-Linear Mech 40(8):1112-1138

2. Bazant Z, Cedolin L (2010) Stability of structures. Elastic, inelastic, fracture and damage theories. World Scientific, London

3. Berry DT, Yang HTY (1996) Formulation and experimental verification of a pneumatic finite element. Int J Numer Methods Eng 39(7):1097-1114

4. Bonet J, Wood RD, Mahaney J, Heywood P (2000) Finite element analysis of air supported membrane structures. Comput Methods Appl Mech Eng 190(5-7):579-595

5. Boyce MC, Arruda EM (2000) Constitutive models of rubber elasticity: a review. Rubber Chem Technol 73(3):504-523

6. Crisfield MA (1990) A consistent co-rotational formulation for non-linear, three-dimensional, beam-elements. Comput Methods Appl Mech Eng 81:131-150

7. Eriksson A (1994) Fold lines for sensitivity analyses in structural instability. Comput Methods Appl Mech Eng 114:77-101

8. Eriksson A (1998) Structural instability analyses based on generalised path-following. Comput Methods Appl Mech Eng 156:4574

9. Eriksson A (2014) Constraint paths in non-linear structural optimization. Comput Struct 140:139-147

10. Eriksson A, Kouhia R (1995) On step size adjustments in structural continuation problems. Comput Struct 55:495-506

11. Eriksson A, Nordmark A (2012) Instability of hyper-elastic balloon-shaped space membranes under pressure loads. Comput Methods Appl Mech Eng 237-240:118-129

12. Geers MGD (1999) Enhanced solution control for physically and geometrically non-linear problems. Part I-the subplane control approach. Int J Numer Methods Eng 46(2):177-204

13. Ghali A, Neville A (1989) Structural analysis. A unified classical and matrix approach, 3rd edn. Chapman and Hall, London 
14. Giannini O, Sestieri A (2016) Experimental characterization of veering crossing and lock-in in simple mechanical systems. Mech Syst Signal Process 72-73:846-864

15. Haslach H, Humphrey J (2004) Dynamics of biological soft tissue and rubber: internally pressurized spherical membranes surrounded by a fluid. Int J Non-Linear Mech 39(3):399-420

16. Haßler M, Schweizerhof K (2008) On the static interaction of fluid and gas loaded multi-chamber systems in large deformation finite element analysis. Comput Methods Appl Mech Eng 197(1920):1725-1749

17. Hill R, Hutchinson J (1975) Bifurcation phenomena in the plane tension test. J Mech Phys Solids 23(4-5):239-264

18. Holzapfel GA (2000) Nonlinear solid mechanics. A Continuum approach for engineering. Wiley, Chichester

19. Ikeda K, Murota K, Fujii H (1991) Bifurcation hierarchy of symmetric structures. Int J Solids Struct 27(12):1551-1573

20. Kearsley E (1986) Asymmetric stretching of a symmetrically loaded elastic sheet. Int J Solids Struct 22(2):111-119

21. Kiousis DE, Gasser TC, Holzapfel GA (2008) Smooth contact strategies with emphasis on the modeling of balloon angioplasty with stenting. Int J Numer Methods Eng 75(7):826-855

22. Koiter W (1970) The stability of elastic equilibrium. Technical Report AFFDL-TR-70-25, Air Force Flight Dynamics Laboratory, Wright-Patterson Air Force Base, Ohio . A translation of the Dutch original from 1945

23. Kolesnikov A (2010) Equilibrium of an elastic spherical shell filled with a heavy fluid under pressure. J Appl Mech Tech Phys 51(5):744-750

24. Lanzoni L, Tarantino A (2014) Damaged hyperelastic membranes. Int J Non-Linear Mech 60:9-22

25. Leissa A (1974) On a curve veering aberration. Zeitschrift für angewandte Mathematik und Physik ZAMP 25(1):99-111

26. Liang DK, Yang DZ, Qi M, Wang WQ (2005) Finite element analysis of the implantation of a balloon-expandable stent in a stenosed artery. Int J Cardiol 104(3):314-318

27. Mooney M (1940) A theory of large elastic deformation. J Appl Phys 11(9):582-592

28. Needleman A (1977) Inflation of spherical rubber balloons. Int J Solids Struct 13(5):409-421

29. Pagitz M, Pellegrino S (2010) Maximally stable lobed balloons. Int J Solids Struct 47(11-12):1496-1507

30. Patil A, DasGupta A (2013) Finite inflation of an initially stretched hyperelastic circular membrane. Eur J Mech A/Solids 41:28-36
31. Patil A, Nordmark A, Eriksson A (2016) Instabilities of wrinkled membranes with pressure loadings. J Mech Phys Solids 94:298315

32. Pipkin A (1986) The relaxed energy density for isotropic elastic membranes. IMA J Appl Math 36:85-99

33. Rao S (1989) The finite element method in engineering, 2nd edn. Pergamon Press, Exeter

34. Rivlin R (1948) Large elastic deformations of isotropic materials. IV. Further developments of the general theory. Philos Trans R Soc Lond A 241:379-397

35. Rodríguez J, Merodio J (2011) A new derivation of the bifurcation conditions of inflated cylindrical membranes of elastic material under axial loading. Application to aneurysm formation. Mech Res Commun 38(3):203-210

36. Roychowdhury S, DasGupta A (2015) On the response and stability of an inflated toroidal membrane under radial loading. Int J NonLinear Mech 77:254-264

37. Rumpel T, Schweizerhof K (2003) Volume-dependent pressure loading and its influence on the stability of structures. Int J Numer Methods Eng 56(2):211-238

38. Steigmann D (1990) Tension-field theory. Proc R Soc Lond A 429:141-173

39. Steigmann DJ (2013) A well-posed finite-strain model for thin elastic sheets with bending stiffness. Math Mech Solids 18(1):103-112

40. Stoop N, Müller MM (2015) Non-linear buckling and symmetry breaking of a soft elastic sheet sliding on a cylindrical substrate. Int J Non-Linear Mech 75:115-122

41. Thompson JMT, Hunt GW (1984) Elastic instability phenomena. Wiley, Chichester

42. Timoshenko SP, Woinowsky-Krieger S (1959) Theory of plates and shells. McGraw-Hill, Kogakusha, Tokyo

43. Zhou Y, Nordmark A, Eriksson A (2015) Instability of thin circular membranes subjected to hydro-static loads. Int J Non-Linear Mech $76: 144-153$

44. Zhou Y, Nordmark A, Eriksson A (2016) Multi-parametric stability investigation for thin spherical membranes filled with gas and fluid. Int J Non-Linear Mech 82:37-48

45. Zienkiewicz OC, Taylor RL (2000) The finite element method: the basis, vol 1, 5th edn. Butterworth-Heinemann, Oxford

46. Zingoni A (2014) Group-theoretic insights on the vibration of symmetric structures in engineering. Philos Trans $\mathrm{R}$ Soc A 372:20120,037 